\title{
Dosimétrie par thermoluminescence
}

\section{Principes de base *}

\author{
D. LAPRAZ et P. IACCONI **
}

(Manuscrit reçu le 20 décembre 1989)

\begin{abstract}
RÉSUMÉ Le but de cet article est de donner un bref aperçu sur les principes de base et une approche théorique de la thermoluminescence. Après une présentation du modèle classique de base et des divers types de recombinaison électron-trou, l'application à la dosimétrie des rayonnements lonisants est développée.
\end{abstract}

ABSTRACT In order to present a short review of the basic principles of, and a theoretical approach to, thermoluminescence, the usual basic thermoluminescence model and the various kinetic electron-hole recombination types are presented. The application to the dosimetry of lonizing radiations is then discussed.

\section{INTRODUCTION}

La thermoluminescence (TL) est l'émission de lumière (fig. 1) observée autour de températures bien définies ("pics"), quand on réchauffe un solide cristallisé (habituellement isolant ou semi-conducteur) qui a préalablement subi une irradiation ionisante provenant du rayonnement naturel (solaire, cosmique, radioéléments) ou artificiel (UV, $X, y, \alpha, \ldots$ ). L'une des propriétés intéressantes de la TL est le fait que son intensité est fonction de la dose absorbée par l'échantillon. C'est un phénomène très différent de l'incandescence qui est régie par la loi de Planck, non seulement par son domaine étendu de température $\left(-273\right.$ à plus de $\left.700^{\circ} \mathrm{C}\right)$, mais aussi par son domaine spectral d'émission (de l'UV au proche infrarouge).

* Communication présentée lors du Séminaire sur la dosimétrie in vivo en radiothérapie et en radlodiagnostic, Nice, 23-24 février 1989.

** Laboratoire d'émission électronique et de luminescence, Université de Nice, parc Valrose, 06034 Nice Cedex, France. 


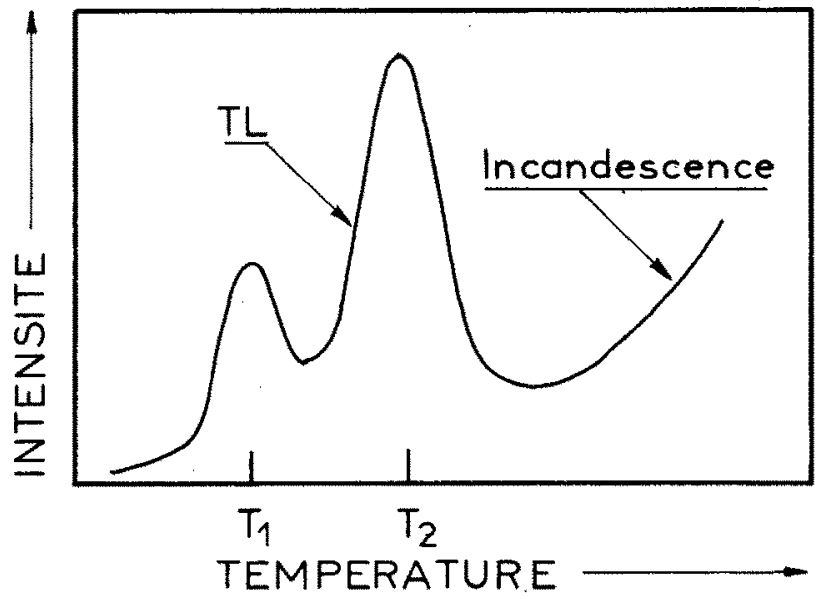

Fig. 1. - Exemple de courbe de thermoluminescence.

Cette émission thermostimulée a été découverte au $17^{e}$ siècle par BOYLE en chauffant un diamant. Ses premiers fondements théoriques ont été élaborés par URBACH [17] puis RANDALL et WILKINS [15] qui donnèrent un schéma simple, utilisé encore dans la plupart des modèles proposés. Le domaine d'application de la $T L$ est vaste, il va de la physique du solide (caractérisation des défauts) à la dosimétrie des rayonnements ionisants (radioprotection, datation archéologique ou géologique $[1,3,6-8,12]$.

Le but de cet article est de donner un bref aperçu des bases et une approche théorique de la TL. Nous aurons affaire essentiellement à des solides cristallisés, ioniques ou ioniques-covalents, et comme tels à des isolants.

\section{Le CRistal ionique parfait, le cristal ioniQue Réel : PRINCIPAUX DÉFAUTS CRISTALLINS}

Un cristal ionique est un arrangement ordonné et alterné d'ions positifs et d'ions négatifs (fig. 2), l'énergie de liaison du cristal (énergie de Madelung) étant régie essentiellement par les interactions électrostatiques (forces de Coulomb). Le cristal ionique parfait est engendré par la répétition rigoureusement périodique d'une maille de forme et de contenu parfaitement définis, la structure cristalline devenant de plus en plus complexe au fur et à mesure que l'ionicité diminue et que le nombre d'ions différents augmente. 
(a)

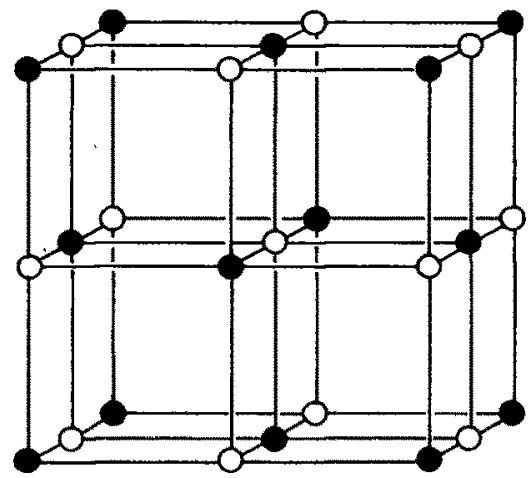

(b)

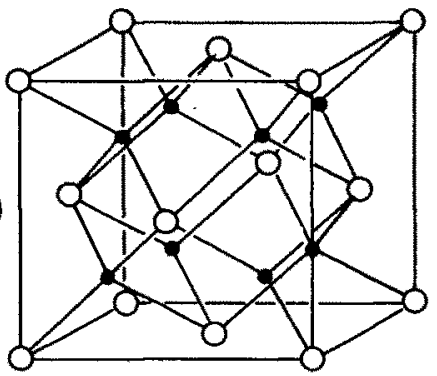

Fig. 2. - Structure : a) du fluorure de lithium (LiF) [0: Li ; $O$; F]

b) de la fluorite $\left(\mathrm{CaF}_{2}[0: \mathrm{Ca} ; \mathrm{O}: \mathrm{F}]\right.$.

En fait, les cristaux réels que nous observons, naturels ou synthétiques, n'ont pas une structure aussi parfaitement définie et contiennent toutes sortes de défauts, qui peuvent jouer un rôle majeur dans toutes les propriétés physiques, notamment dans le processus de TL. Les défauts cristallins les plus importants sont de deux types:

\section{Les défauts ponctuels :}

- Lacunes (absence d'ions + ou d'ions -) ; ces lacunes ne doivent pas être confondues avec celles qui résultent d'un effet thermique (défauts de Schottky ou de Frenkel) conduisant à la diffusion des lons dans un cristal, aussi parfait soit-il (conductivité ionique) ; les lacunes sont la cause de la grande diversité de centres pouvant piéger des électrons ou des trous, d'où l'existence de centres à électrons et de centres à trous pouvant donner une coloration au cristal (centres colorés) : l'un des plus importants centres à électrons dans les halogénures alcalins est le centre $F$, constitué d'un électron plégé dans une lacune anionique (la figure 3 donne la structure des centres les plus usuels dans les halogénures alcalins et qui ont fait l'objet d'un très grand nombre d'études en physique du solide) ;

- ions interstitiels : ce sont des ions du cristal qui ne se trouvent pas dans leur site normal ;

- impuretés chimiques (ions étrangers au réseau) ; elles peuvent se trouver en position soit interstitielle, soit substitutionnelle (exemple : $\mathrm{Mn}^{2+}$ à la place de $\mathrm{Ca}^{2+}$ dans $\left(\mathrm{CaF}_{2}\right)$;

2. Les défauts structuraux : dislocations, fautes d'empilement, macles, défauts de surface et de volume... 


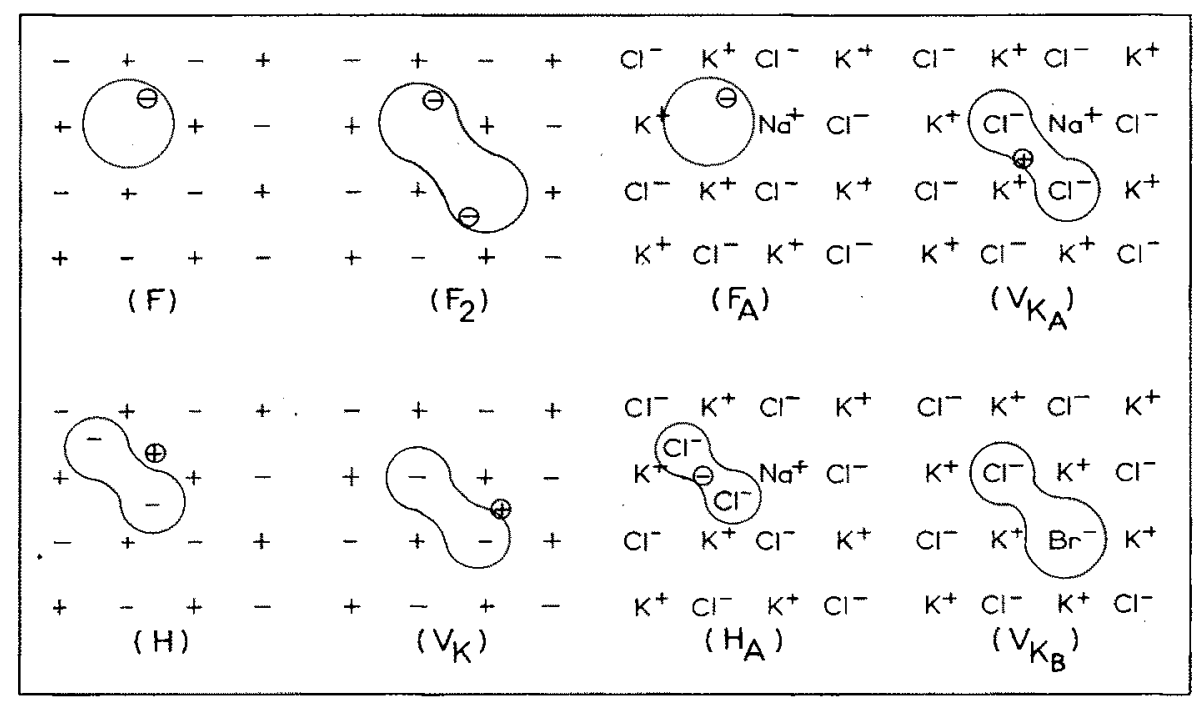

Fig. 3. - Quelques centres colorés dans les halogénures alcalins, d'après FARGE ot FONTANA [4].

\section{DIAGRAMME DANS LE CADRE DE LA REPRÉSENTATION DE LA THÉORIE DES BANDES D'ÉNERGIE : CENTRES DE PIÉGEAGE ET CENTRES DE RECOMBINAISON (RADIATIVE OU NON)}

La structure périodique du réseau d'un cristal parfait entraîne que le potentiel cristallin, dans lequel se trouvent les électrons, varie périodiquement (puits de potentiel). Dans un tel milieu, on montre que les énergies des électrons peuvent être regroupées dans des bandes permises (répartition quasi continue) séparées par des bandes interdites de grande largeur (théorie des bandes d'énergie).

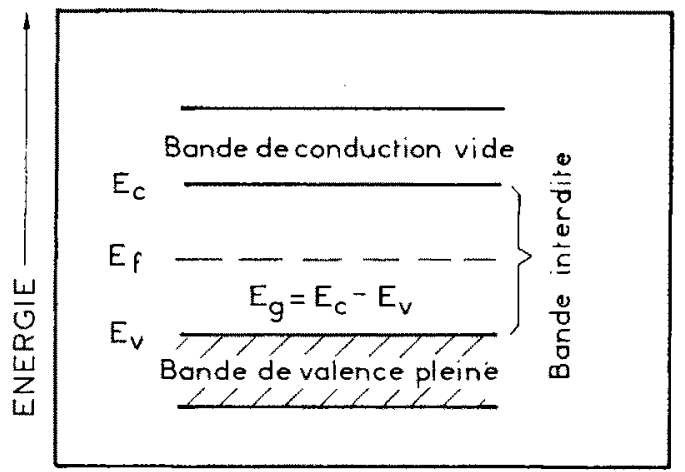

Fig. 4. - Bandes d'énergie dans le cas d'un isolant. 
La bande supérieure d'énergies permises est appelée bande de conduction $(B C)$. A O K, elle est vide ; toutes les autres bandes permises sont intégralement remplies d'électrons. La bande pleine supérieure est appelée bande de valence (BV). Ces deux bandes sont séparées par une bande interdite (BI) dont la largeur énergétique est appelée gap (fig. 4). Le gap dans le cas des isolants est habituellement superieur à $6 \mathrm{eV} ; \mathrm{ceci}$ explique que le passage d'un électron de la bande de valence à la bande de conduction ne peut se faire qu'avec des excitations de forte énergie (dites ionisantes).

La présence de défauts se traduit par l'introduction de niveaux d'énergie permis dans la bande interdite; deux types de niveaux sont ainsi introduits :

- des centres de piégeage (ou pièges),

- des centres de recombinaison.

La figure 5 montre les niveaux d'énergie d'un isolant à l'équilibre à $0 \mathrm{~K}$ : tous les niveaux au-dessous de $E_{f}$ (énergie de Fermi) sont remplis d'électrons alors que ceux qui sont au-dessus sont vides.

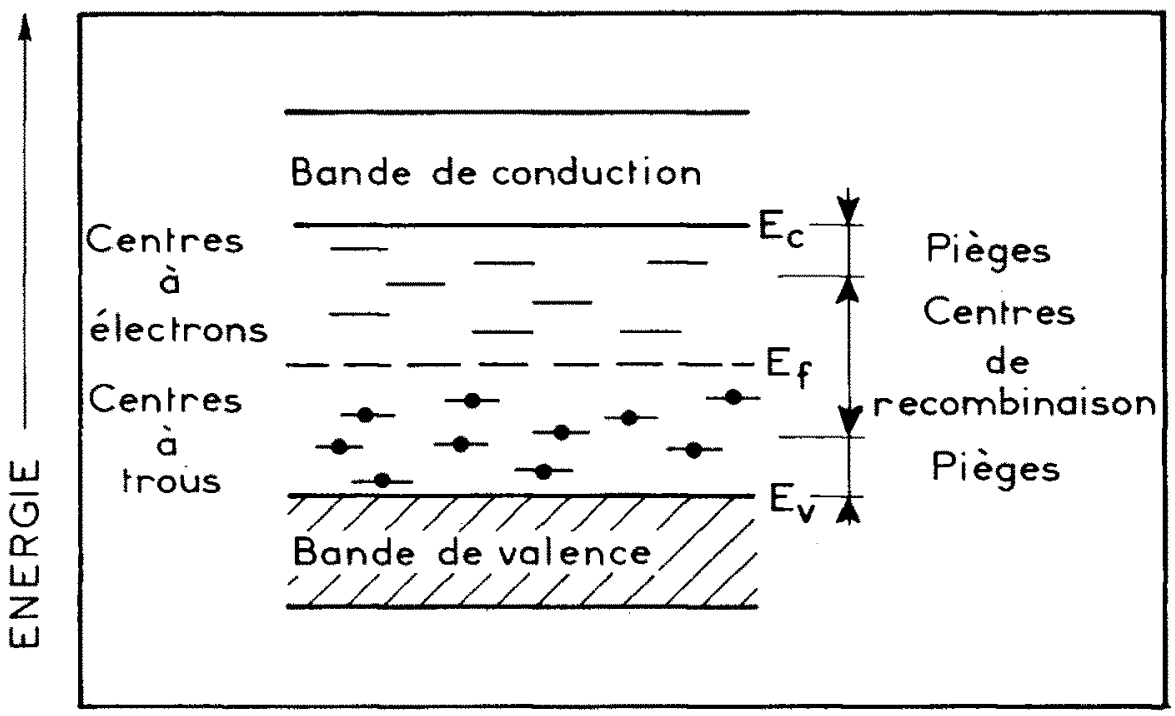

Flg. 5. - Niveaux d'énergle d'un lsolant a l'équillibre $(T=0 \mathrm{~K})$, d'apròs McKeEVER [7].

Les pièges à électrons (ou à trous), centres susceptibles de capturer des électrons (ou trous), sont situés près de la bande de conduction (bande de valence) à une distance énergétique inférieure à $2-3 \mathrm{eV}$, alors que les centres de recombinaison sont généralement situés au-delà. 
La distinction entre pièges et centres de recombinaison repose essentiellement sur les probabilités de capture des porteurs de charges. Si $\sigma_{e}$ est la section efficace de capture d'un électron et $\sigma_{t}$ est celle de capture d'un trou :

1. un piège à électron (piège à trou) est caractérisé par le fait que $\sigma_{e} \gg \sigma_{t}\left(\sigma_{e}<<\sigma_{t}\right)$; c'est un niveau dit métastable, ce qui veut dire qu'il faudra fournir une énergie dite d'activation $E$, appelée profondeur de piège, qui correspond généralement à la distance énergétique entre le piège et le bas de la bande de conduction (le haut de la bande de valence) pour délocaliser l'électron (trou) qui aurait été capturé sur ce centre ;

2. un centre de recombinaison est caractérisé par le fait que $\sigma_{e} \approx \sigma_{t}$ avec $\sigma_{e}$ et $\sigma_{t}$ relativement importants. La recombinaison électron-trou peut se faire ;

- soit radiativement, on dira alors qu'on a affaire à un centre de luminescence; dans le cas où ce dernier est associé à une impureté, celle-ci sera appelée activateur, par exemple $\mathrm{Mn}$ et Dy dans $\mathrm{CaF}_{2}$, ou $\mathrm{Cu}$ dans $\mathrm{Li}_{2} \mathrm{~B}_{4} \mathrm{O}_{7}$,

- soit non radiativement, on dira qu'on a affaire à un centre "poison" ; l'impureté associée est appelée inhibiteur : par exemple Fe dans la plupart des cristaux.

D'une manière générale, pour chaque centre de recombinalson, il faut savoir que le rendement de luminescence

$$
n=\frac{P_{r}}{P_{r}+P_{n r}}
$$

où $P_{r}$ et $P_{n r}$ sont respectivement les probabilités de recombinaison radiative et non radiative, varie avec la température et s'annule au-delà d'une certaine température : c'est l'extinction thermique de la luminescence ; par exemple l'émission du centre $\mathrm{F}^{+}$à $320 \mathrm{~nm}$ de l'alumine disparaît au-delà de $70-120^{\circ} \mathrm{C}$, ce qui explique qu'elle ne soit plus observée dans l'émission du pic dosimétrique à $240^{\circ} \mathrm{C}$.

\section{LA THERMOLUMINESCENCE : MODÉLE DE BASE ; APPROCHE QUANTITATIVE}

\subsection{Modèle de base}

La phénoménologie de la TL est fondée sur plusieurs modèles. Nous considérerons ici le modèle le plus simple à deux niveaux dans la bande interdite (fig. 6) :

- un piège à électrons $T$ situé au-dessous de la bande de conduction à une profondeur d'énergie $E$,

- un centre de recombinaison $R$. 


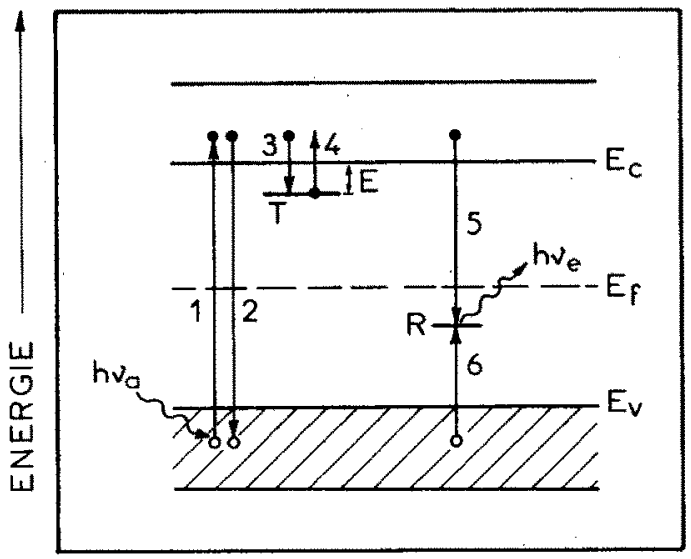

Fif. 6. - Modèle simple à deux niveaux avec les transitions permises suivantes : (1) Ionisation, (2) retour à l'état fondamental, (3) et (6) piégeage,

(4) déplégeage thermique, (5) recombinalson radiative.

Initialement $(t=0)$, nous supposerons que le piège est vide. Nous pouvons diviser en deux étapes la façon dont un matériau acquiert une thermoluminescence quand il est soumis à une irradiation ionisante :

1. ionisation et piégeage d'électrons au cours de lirradiation du corps (absorption d'énergie),

2. recombinaison électron-trou avec émission radiative par chauffage du matériau (restitution d'énergie).

Pendant l'irradiation, il y a absorption d'énergie $\mathrm{hv}_{\mathrm{a}}$ dans le cristal et processus d'ionisation (transition 1) faisant passer un électron de la bande de valence à la bande de conduction ; l'électron laisse ainsi un trou dans la bande de valence qui viendra se piéger sur $\mathbf{R}$ (transition 6). L'électron peut :

- soit se recombiner tout de suite $C_{0} \approx 10^{-8}$ à $10^{-10} \mathrm{~s}, \tau_{0}$ étant la durée de recombinaison, relativement indépendante de la température) avec le trou (processus 2) ; si la recombinaison est radiative, on aura affaire à une fluorescence, caractéristique de la matrice cristalline ;

- soit se recombiner avec le centre de recombinaison $R$ (processus 5) qui a capté un trou, donnant une émission radiative d'énergie $h_{\mathrm{e}}$ caractérisant l'activateur ;

- soit tomber dans le piège $T$ (processus 3) ; la transition du niveau piège vers le centre de recombinaison étant interdite, l'électron restera piégé pendant une durée de vie telle que :

$$
\frac{1}{\tau}=p=s \exp \left(\frac{-E}{k T}\right)
$$


avec :

p = probabilité de dépiégeage par unité de temps (s)

$s=$ facteur de fréquence (en général compris entre $10^{6}$ et $10^{18} \mathrm{~s}^{-1}$ )

$\mathrm{E}=$ profondeur du piège (habituellement en $\mathrm{eV}$ )

$\mathrm{k}=$ constante de Boltzmann $=0,86167.10^{-4} \mathrm{eV} / \mathrm{K}$

$\mathrm{T}=$ température du cristal (K).

La profondeur du piège $E$ apparaît donc comme une énergie d'activation nécessaire pour faire passer l'électron du niveau métastable vers un niveau supérieur (ici la bande de valence) (processus 4) à partir duquel le retour à l'état initial est permis (processus 2):

- Si E est faible (piège peu profond) l'agitation thermique du réseau peut suffire pour communiquer à l'électron piégé l'énergie nécessaire à son dépiégeage. Son transfert au centre de recombinaison $R$ donnera lieu à une émission appelée phosphorescence. A température ambiante, ce sont des pièges dont la profondeur est de l'ordre de (ou inférieure à) $0,6 \mathrm{eV}$ qui donnent lieu à la phosphorescence $(\approx 1 \mathrm{~s}$ à plusieurs jours, cette durée de recombinaison étant très dépendante de la température comme le montre la relation (1)).

- Si $E$ est relativement important, le déplégeage de l'électron sera accéléré par apport d'énergie thermique extérieure. L'émission observée est alors appelée thermoluminescence et le mécanisme est le même que ci-dessus : ainsi, la TL peut être considérée comme une phosphorescence activée thermiquement. Habituellement, on élève progressivement la température du cristal suivant une loi linéaire en fonction du temps.

L'enregistrement des variations de l'émission lumineuse en fonction de la température donne la courbe de thermoluminescence (ou thermoluminogramme) (fig. 1). Dans le cas d'un piège unique, la courbe est constituée d'un seul pic, caractérisé par la température $T^{*}$ de son maximum et par sa forme ; c'est à partir de cette courbe que l'on définit trois paramètres caractérisant le piège : la profondeur $(E)$, le facteur de fréquence (s) et l'ordre de la cinétique (I).

Une autre information importante est lanalyse spectrale du pic de TL, qui permettra de déterminer l'énergie émise lors de la recombinaison électron-trou, correspondant à la distance énergétique qui sépare le centre $R$ du bas de la bande de conduction (fig. 6). Habituellement, on utilise des produits dont l'émission de TL est située dans le domaine visible ou proche UV:

\subsection{Approche quantitative}

Après s'être dépiégé, l'électron peut éventuellement être recapturé par le piège. Si on appelle q la probabilité de repiégeage et $r$ la probabilité de recombinaison (fig. 7), on peut calculer la variation de l'intensité de $T L$ en fonction de la température dans des cas simples. 


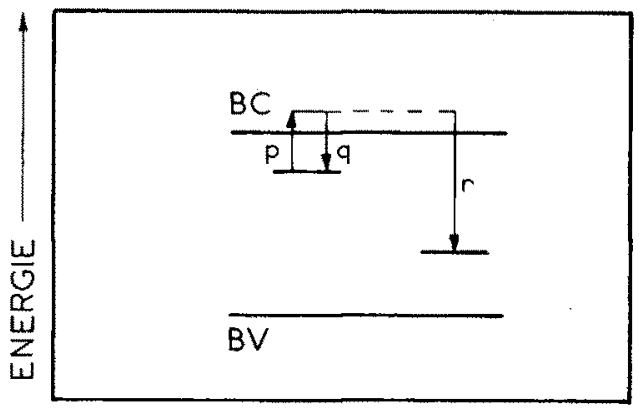

Fig. 7. - Définitions des probabilités de piégeage $p$, de dépiégeage $q$ et de recombinaison $r$.

\section{Supposons :}

1) que la vitesse de chauffage de l'échantillon $\beta=\frac{d T}{d t}$ soit constante,

2) qu'il y ait un seul type de pièges et que tous ces pièges soient pleins à l'instant initial $(t=0)$,

3) que la durée de vie des électrons dans la bande de conduction soit faible,

4) qu'il n'y ait pas d'extinction thermique de l'émission.

On considérera 3 types de cinétiques : deux particulières et une générale.

a - Cinétique du premier ordre $(l=1)$

Si à l'instant $t$, la température du cristal est $T$ et s'il y a $n$ électrons dans le piège, alors la vitesse de sortie des électrons du piège est :

$$
-\frac{d n}{d t}=n p=n s \exp \left(-\frac{E}{k T}\right)
$$

Dans le cas où il n'y a pas de repiégeage $(q=0)$, la cinétique sera dite du premier ordre. C'est le modèle de RANDALL et WILKINS [2]. Alors l'intensité de luminescence est donnée par :

$$
I=-C \frac{d n}{d t}=C n s \exp \left(-\frac{E}{k T}\right)
$$

où $C$ est un facteur de proportionnalité lié au rendement de luminescence.

- A T constant, d'après la relation (2), on en tire :

$$
\mathrm{n}=\mathrm{n}_{0} \exp (-\mathrm{pt})
$$

d'où un déclin de phosphorescence exponentiel :

$$
I=I_{0} \exp (-p t)
$$


Si l'échantillon est chauffé à vitesse constante $\beta$ :

$$
\frac{d n}{d t}=\beta \frac{d n}{d T}
$$

et en remplaçant dans l'expression (2) :

$$
\frac{d n}{n}=-\frac{s}{\beta} \exp \left(-\frac{E}{k T}\right) d T
$$

d'où :

$$
n=n_{0} \exp \left(-\int_{T_{0}}^{T} \frac{s}{\beta} \exp \left(-\frac{E}{k T}\right) d T\right)
$$

où $n_{0}$ est le nombre d'électrons présents dans le piège à l'instant $t=0$ et à la température $T_{0}$.

Par conséquent, en reportant l'expression (8) dans (3), l'intensité de TL sera donnée par :

$$
I=C n_{0} s \exp \left(-\frac{E}{k T}\right) \cdot \exp \left(-\frac{s}{\beta} \int_{T_{0}}^{T} \exp \left(-\frac{E}{k T}\right) d T\right)
$$

En traçant I en fonction de $T$, on obtient bien un pic de TL schématisé sur la figure 8.

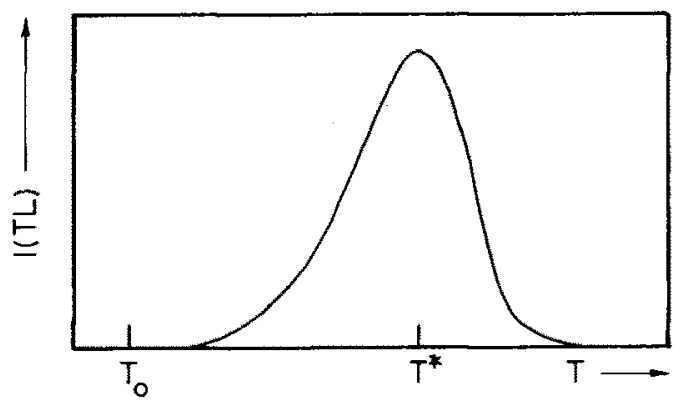

Fig. 8. - Allure d'un pic de TL dans le cas d'une cinétique du premier ordre. 
b - Cinétique du second ordre $(I=2)$

Dans le cas où la probabilité de repiégeage est du même ordre de grandeur que celle de recombinaison, $q \approx r$ (cela est aussi vrai si $q \gg r$ ), la cinétique de recombinaison est dite du second ordre. GARLICK et GIBSON [10] ont montré que, daris ce cas :

$$
I=\frac{n_{0} s \exp \left(-\frac{E}{k T}\right)}{\left[1+\frac{s}{\beta} \int_{T_{0}}^{T} \exp \left(-\frac{E}{k T}\right) d T\right]^{2}}
$$

Le déclin de la phosphorescence est alors hyperbolique :

$$
I=\frac{I_{o}}{\left(t+t_{0}\right)^{2}}
$$

$t_{0}$ étant un paramètre.

c - Cinétique d'ordre quelconque

En réalité, dans beaucoup de cas, le déclin correspond plutôt à la formule de BECQUEREL :

$$
I=\frac{I_{0}}{\left(t+t_{0}\right)^{\alpha}}
$$

$\alpha$ étant un nombre fractionnaire, compris en général entre 1 et 2 et relié à l'ordre de la cinétique 1 par :

$$
1=\frac{\alpha}{\alpha-1}
$$

MAY et PARTRIDGE [11] ont montré que l'intensité de la TL pouvait s'écrire :

$$
I=C n_{0} s \exp \left(-\frac{E}{k T}\right) \cdot\left[\frac{(1-1) s}{\beta} \int_{T_{0}}^{T} \exp \left(-\frac{E}{k T}\right) d T+1\right]-\frac{1}{1-1}
$$

L'utilisation de ces formules permet de déterminer les valeurs des paramètres $E$, $s$ et 1 . 


\subsection{Principe de mesure d'une dose d'irradiation}

La théorie montre, en partant de la relation (3), que la surface du pic de $T L$ (et avec une bonne approximation, son intensité maximale) est directement liée au nombre $n_{0}$ d'électrons piégés, c'est-à-dire à la dose absorbée dans l'échantillon. C'est cette propriété qui est utilisée en dosimétrie par TL. Le dispositif classique de mesure de TL devra comporter (fig. 9), un dispositif chauffant avec mesure de température, un dispositif de détection de la TL et d'enregistrement des courbes de TL couplé à un système intégrateur donnant la surface du pic.

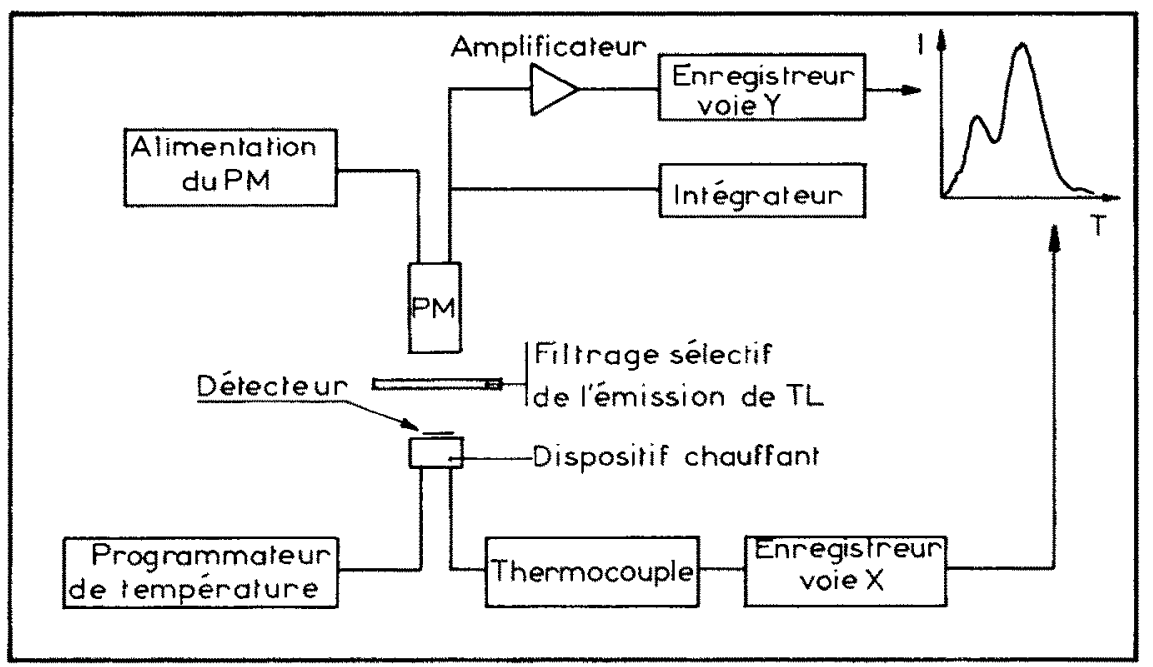

Fig. 9. - Schéma simplifié d'un lecteur de, dosimétrie par TL.

Une telle description est très schématique ; disons simplement que les qualités requises pour un tel lecteur sont en particulier les suivantes : précision et reproductibilité de la mesure, rapidité de la lecture, fiabilité, prix peu élevé.

\subsection{Conclusion}

En réalité, les choses ne sont pas aussi simples et la description quantique d'un matériau thermoluminescent fait souvent intervenir plusieurs types de pièges et de centres de recombinaison auxquels correspondent plusieurs pics de TL. Lorsqu'on chauffe un tel échantillon, les pièges se vident les uns après les autres en commençant par les moins profonds. C'est ainsi, par exemple, que la courbe de TL du fluorure de lithium (LiF) couramment utilisé en dosimétrie présente au moins 6 pics enchevêtrés (fig. 10). 


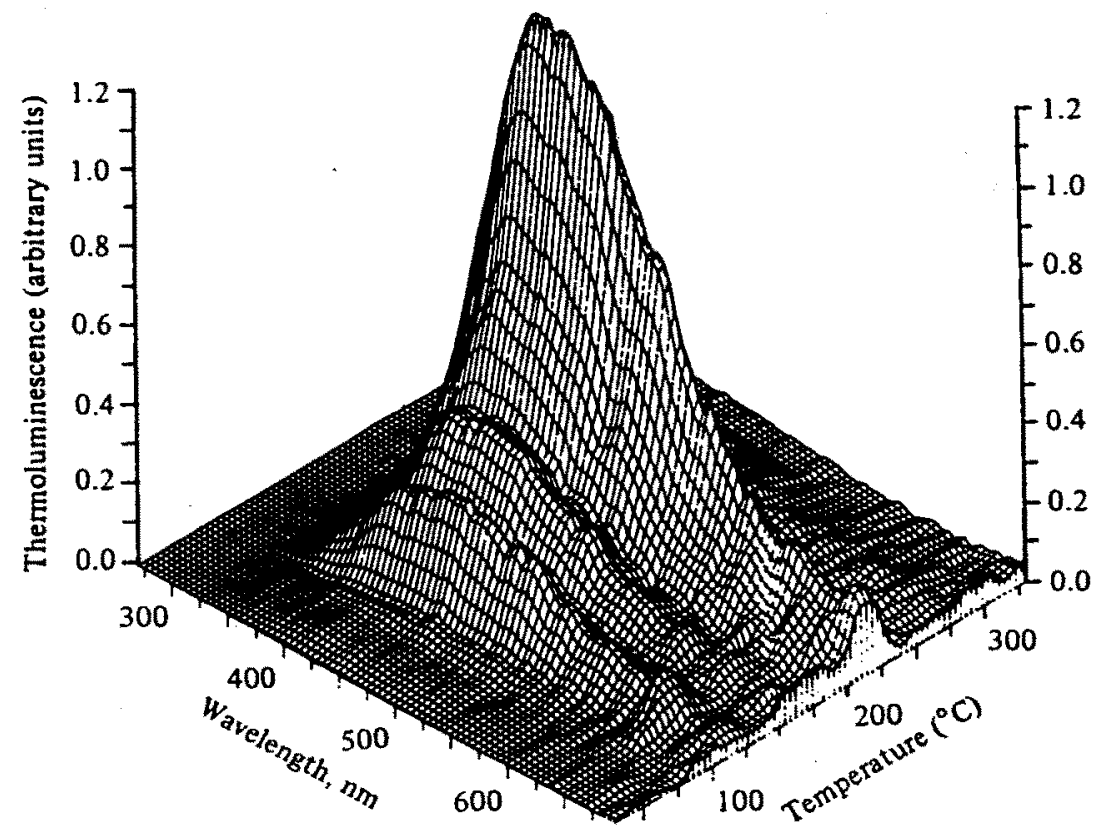

Fig. 10. - Courbe de TL, avec analyse spectrale, de LIF (TLD 600) Irradié aux rayons $X$, d'après TOWNSEND ot al. [16].

Le modèle proposé par MAYHUGH [13] pour l'interprétation de ces pics fait intervenir différents types de centres : $F, V_{3}$ et $V_{K}$. De plus, du point de vue dosimétrique, tous les pics n'ont pas le même comportement : seuls, certains sont utilisables en dosimétrie. Leur proximité ainsi que le comportement spécifique du matériau imposent donc l'adoption de techniques appropriées (recuit préalable...).

\section{PRINCIPALES PROPRIÉTÉs dE QUELQUES MATÉRIAUX THERMOLUMINESCENTS UTILISÉS EN DOSIMÉTRIE}

Les propriétés des corps thermoluminescents utilisés en dosimétrie sont caractérisés par plusieurs paramètres tels que :

- forte sensibilité de détection $(X, \gamma, \alpha, n, \ldots)$ et ceci avec de très faibles quantités de matières (quelques milligrammes) enfermés dans des petites capsules étanches de téflon ;

- domaine spectral d'émission : si possible à $\lambda<600 \mathrm{~nm}$, c'est-à-dire loin du spectre d'émission d'incandescence afin d'avoir un bon rapport signal/bruit de fond (fig. 11) ; 


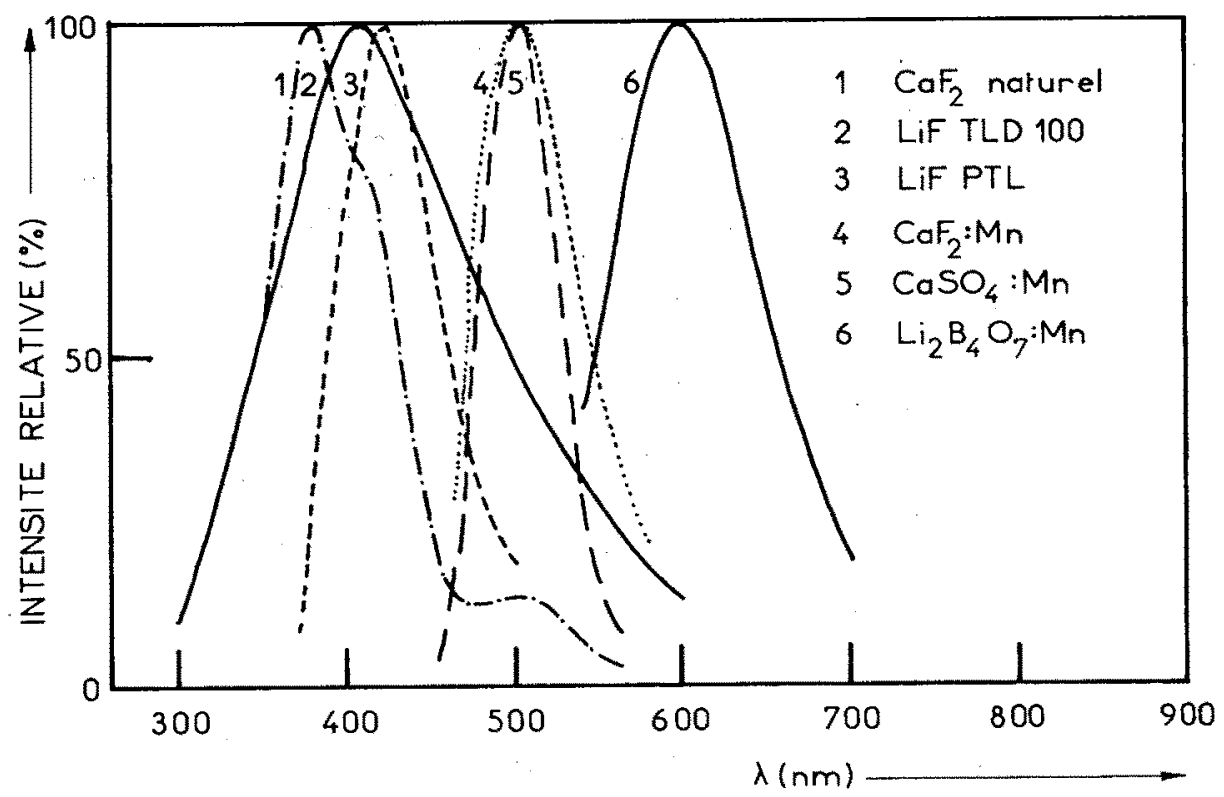

Fig. 11. - Spectres d'émission de TL de quelques prodults dosimétriques, d'après PORTAL [13].

- stabilité de l'information : on utilise des pics situés à $T \geq 200^{\circ} \mathrm{C}$ qui ont un faible affaiblissement thermique et on s'assurera que ces pics ne sont pas sensibles à la lumière ambiante (affaiblissement optique) ;

- linéarité de la réponse en fonction de la dose : sur la figure 12 est reportée la courbe de réponse du LiF en fonction de la dose absorbée. En général, on distingue trois zones : une zone linéaire, une zone dite supralinéaire, une zone de saturation ; d'une façon générale, c'est la zone linéaire qui est utilisée, là où la réponse est directement proportionnelle à la dose absorbée ;

- réponse en fonction de l'énergie et du type de rayonnement; la figure 13 montre, par exemple, comment varie la réponse de divers matériaux thermoluminescents en fonction de l'énergie du rayonnement électromagnétique $(X, \gamma)$;

- influences diverses : température pendant et après l'irradiation, recuit thermique, débit de dose, milieu ambiant (humidité,...), action de la lumière, (UV...), etc.

Pour terminer, le tableau I présente quelques caractéristiques de produits thermoluminescents couramment utilisés en dosimétrie. 
DOSIMETRIE PAR THERMOLUMINESCENCE

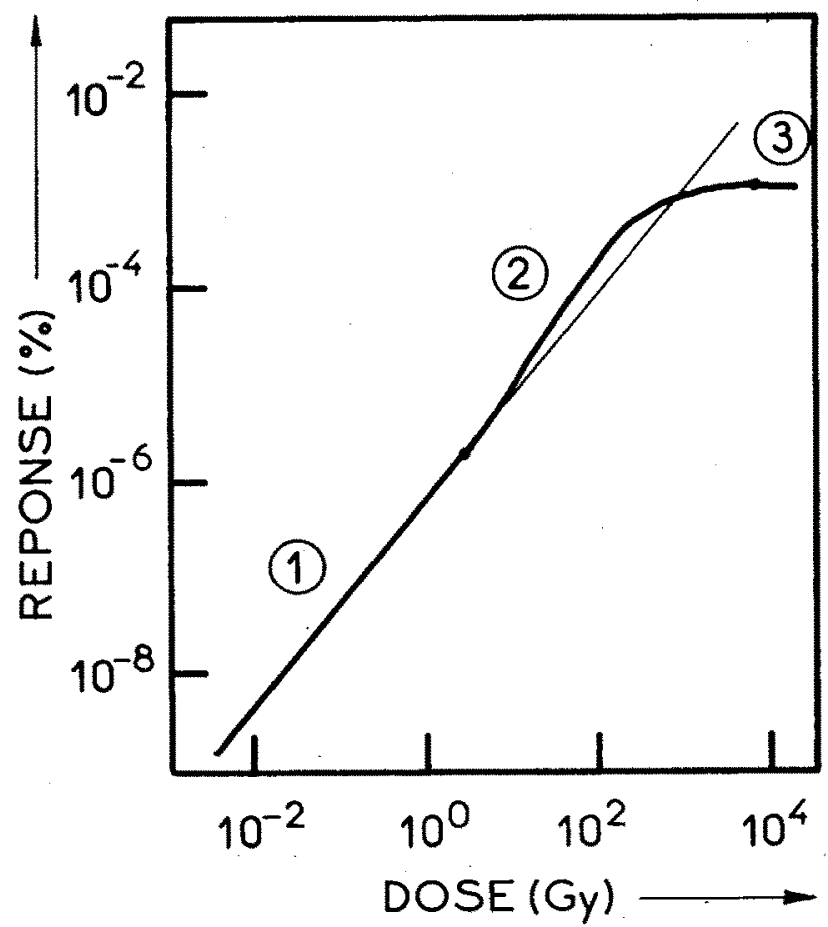

Fig. 12. - Courbe de réponse du Lif en fonction de la dose absorbée, d'apres MARRONE et ATTIX [9].

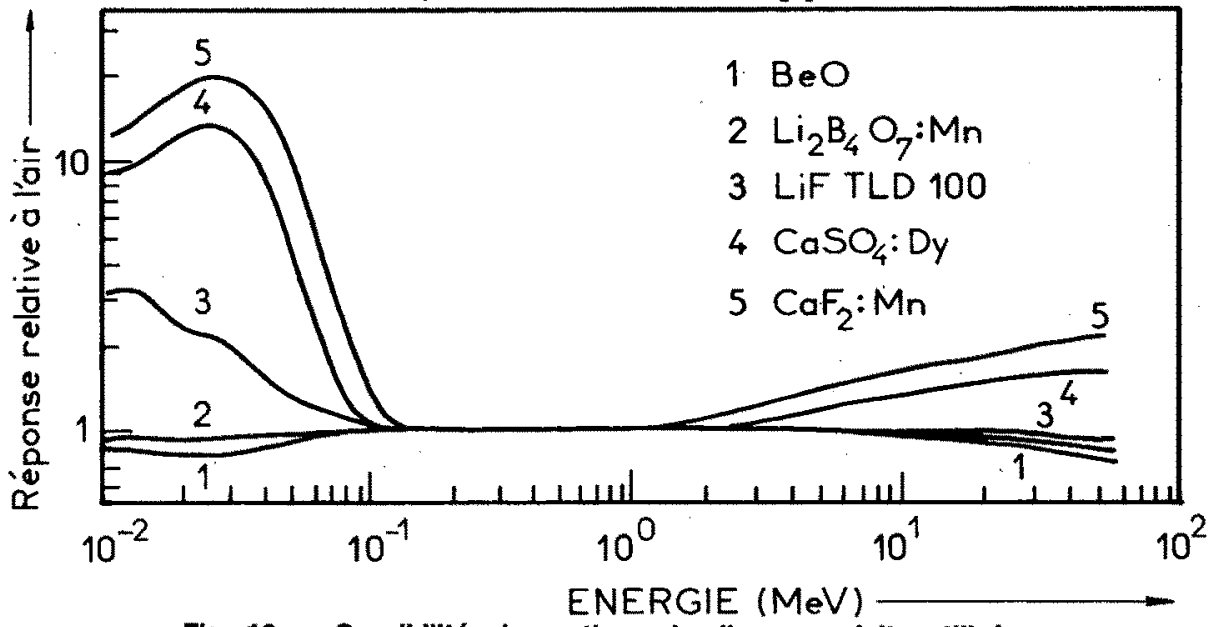

Fig. 13. - Sensibilite chromatique de divers produite utllisés en dosimétrie par TL relative à une dose absorbée dans l'air de $10 \mathrm{mGy}\left({ }^{60} \mathrm{Co}\right)$, d'après BUSUOLI [2]. 
D. LAPRAZ - P. IACCONI

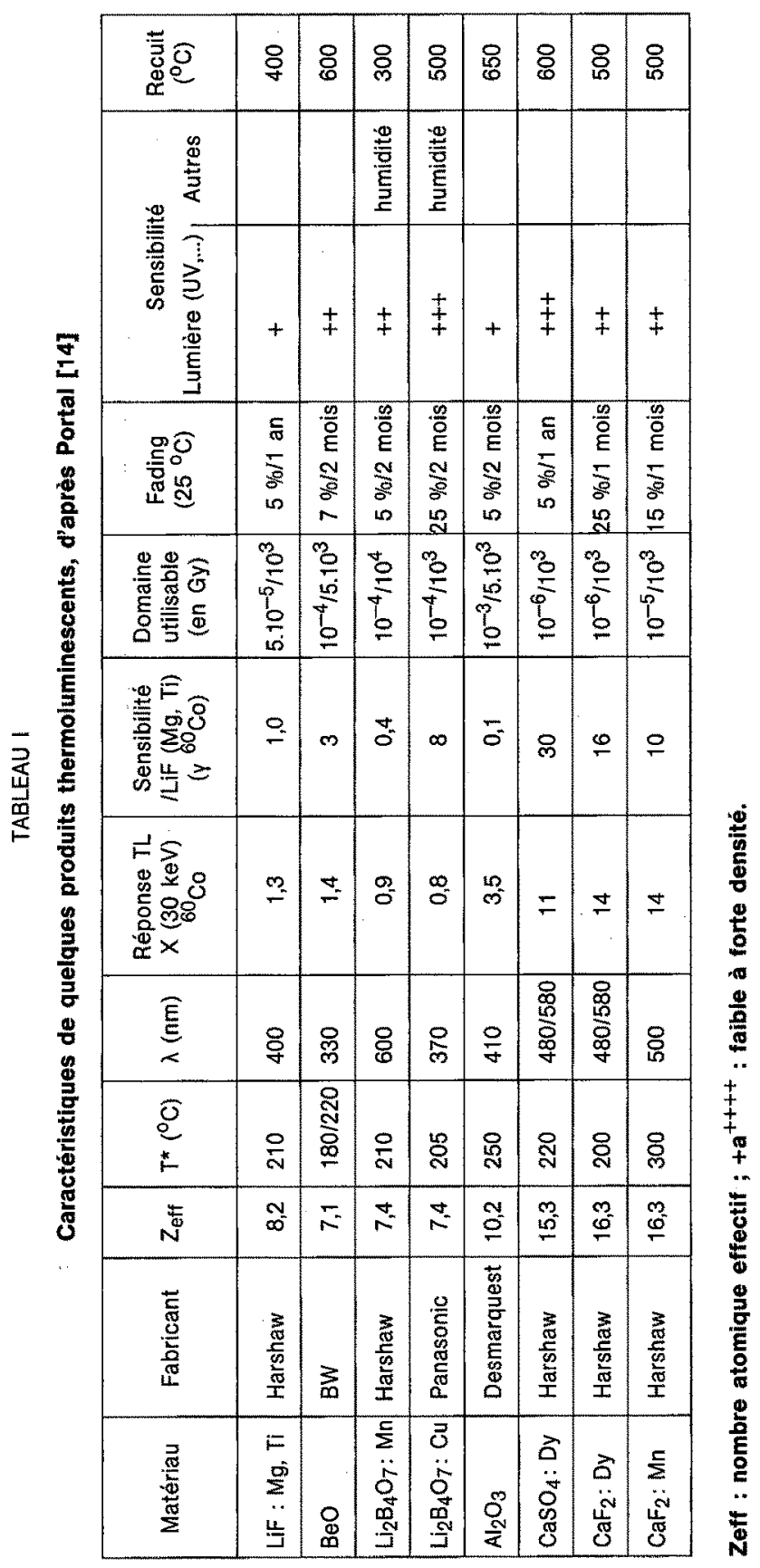




\section{BIBLIOGRAPHIE}

[1] AITKEN M.J. - Thermoluminescence dating. London : Academic press, 1985.

[2] BUSUOLI G., cité par PORTAL G. - Thèse, Université Paul Sabatier, Toulouse, 1978.

[3] CHEN R. et KIRSH Y. - Analysis of thermally stimulated processes.

[4] FARGE $Y$. et FONTANA M. - Perturbations électroniques et vibrationnelles localisées dans les solides ioniques. Paris : Masson, 1974.

[5] GARLICK G.F.J. et GIBSON A.F. - Proc. Phys. Soc., 1948, 60, 574-590.

[6] McDOUGALL D.J. - Thermoluminescence of geological materials. London : Academic press, 1968.

[7] McKEEVER S.W.S. - Thermoluminescence of solids. Cambridge : University press, 1985.

[8] McKINLAY A.F. - Thermoluminescence dosimetry. Bristol : Adam Hilger, 1981.

[9] MARRONE M.J. et ATTIX F.H. - Health Phys., 1964, 10, 431.

[10] MAY C.E. et PARTRIDGE J.A. - J. Chem. Phys., 1964, 40, 1401-1409.

[11] MAYHUGH M.R. - J. Appl. Phys., 1970, 41, 4776.

[12] OBERHOFER $M$. et SCHARMANN A. - Eds. Applied thermoluminescence dosimetry, Bristol : Adam Hilger, 1979.

[13] PORTAL G. - voir réf. [12], 97-122.

[14] PORTAL G. - Radiat. Prot. Dosim., 1986, 17 (1-4) 351-357.

[15] RANDALL J.T. et WILKINS M.H.F. - Proc. Roy. Soc., 1945, A184, 365-407.

[16] TOWSEND P.D., AHMED K., CHANDLER P.J., MCKEEVER S.W.S. et WHITLOW H.J. - Radiat. Eff., 1983, 72, 245.

[17] URBACH F., SITZBER - Akad. Wiss. Wein. Math., Nature, 1930, 139, 363.72. 


\section{LISTE DES ACTES DISPONIBLES AU SECRÉTARIAT DE LA SFRP}

- Les concepts de faible dose de rayonnements ionisants en radiobiologie et en radioprotection, 29-30 avr. 1975 - $60 \mathrm{~F}$.

- Colloque sur les implications psycho-sociologiques du développement de l'industrie nucléaire, 13-15 janv. 1977 - $100 \mathrm{~F}$.

- Effets biologiques des rayonnements non kionisants - utilisation et risques associés, 22-26 mai 1978 - $150 \mathrm{~F}$.

- Journées d'étude sur les dispositions prises à la conception et en exploitation pour réduire la radioexposition professionnelle dans les centrales nucléaires à eau ordinaire, $12-14$ déc. $1979-180 \mathrm{~F}$.

- Séminaire sur les déchets radioactifs, 2-6 févr. 1981 - $150 \mathrm{~F}$.

- Impact radiologique des centrales et autres installations nucléaires sur l'homme et son environnement, 30 sept.-2 oct. 1981 - $150 \mathrm{~F}$.

- Protection au cours de l'utilisation des rayonnements ionisants et non ionisants en médecine, 14-16 déc. 1981 - $150 \mathrm{~F}$.

- Risques liés à l'utilisation du laser, 21 janv. 1982 - 80 F.

- Les sources radioactives, 16 fév. 1982 - $90 \mathrm{~F}$.

- La ventilation des installations, 22-26 mars 1982 - $150 \mathrm{~F}$.

- Comparaison des risques associés aux grandes activités humaines, 18-22 oct. - $200 \mathrm{~F}$.

- Journées d'études "plutonium et radioprotection", 14-16 juin 1983 - $150 \mathrm{~F}$.

- Introduction à la radioprotection, 1985 - $200 \mathrm{~F}$.

- Les expositions professionnelles - Aspects méthodologiques et expériences pratiques, 29-31 janv. $1986-200 \mathrm{~F}$.

- Journées "Tritium", 23-25 avr. 1986 - $200 \mathrm{~F}$.

- La maîtrise d'un système de transport de matières dangereuses: le cas des matières radioactives, 7-8 oct. 1986 - $200 \mathrm{~F}$.

- Conséquences médicales de laccident nucléaire de Tchernobyl, 14-15 mai 1987 - $300 \mathrm{~F}$.

- Ergonomie et protection, 12 mars 1987 - $150 \mathrm{~F}$.

- Mesures dans l'environnement et impact sanitaire à la suite de l'accident nucléaire de Tchernobyl, 12-13 oct. 1987 - $200 \mathrm{~F}$.

- Modification de l'action biologique des rayonnements ionisants radioprotecteurs et radiosensibilisateurs, 22 nov. $1988=150 \mathrm{~F}$.

- Exposition au radon dans les habitations: aspects techniques et sanitaires, 10-11 janv. $1989-200 \mathrm{~F}$.

\section{ACTES DISPONIBLES A LA SFRP SUD-EST}

- Impact dans l'environnement des centrales électronucléaires - Programmes de recherches EDF/CEA en radioécologie, 2 juin 1987.

- Journées uranium, 10-11-12-13 oct. 88 . 


\title{
Technologie des lecteurs de dosimètres thermoluminescents *
}

\author{
Jean BARTHE et Guy PORTAL **
}

(Manuscrit reçu le 22 mai 1989)

RÉSUMÉ La dosimétrie par matériaux thermoluminescents a pris un essor considérable ces dernières années tant en radioprotection (dosimétrie légale dans de nombreux pays) qu'en physique radiologique pour le suivi des irradiations en radiothérapie. Si la conception d'un lecteur pour la radioprotection est relativement aisée compte tenu de l'erreur admise en particulier aux faibles doses $( \pm 10 \%)$, il en va tout autrement en physique radiologique lorsque l'erreur maximale tolérée est d'environ $1 \%$. Les choix technologiques et leur adéquation avec les dosimètres utilisés constituent le "secret" de la qualité des performances de l'ensemble dosimétrique citons en particulier : le principe de la stimulation thermique ou optique, le type de photomultiplicateur et son mode de fonctionnement, le filtrage optique et les méthodes d'intégration du signal. Des tableaux donnent les références et les caractéristiques des principaux lecteurs commerciaux ou commercialisables. Quatre lecteurs possédant des méthodes originales et peu courantes sont décrits de façon plus précise. La conception de matérlaux organiques optostimulables par laser de puissance dans le proche infrarouge est envisagée.

La dosimétrìe par thermoluminescence restera pendant de nombreuses années une technique de choix pour la dosimétrie en radiothérapie. Avec un matériau détecteur comme le borate de lithium activé au cuivre, équivalent tissu presque parfait, et les nouveaux lecteurs automatiques, la qualité, la reproductibilité et la rapidité d'obtention des résultats sont un atout important dans le suivi dosimétrique du patient.

ABSTRACT A considerable increase has occured over recent years in the use of thermoluminescent materials for radiation protection (as a legal method of dosimetry in many countries) and for monitoring radiotherapeutic exposures. Whereas it is relatively easy to design readers for radiation protection purposes, measurement accuracies of $\pm 10 \%$ being acceptable particularly for low doses, the situation is quite different in radiological physics where the maximum tolerable error is of the order of $1 \%$. The overall quality of the results will depend on the technology employed once the appropriate choice of the dosimetric material has been made. Different technological trade offs include thermal or optical stimulation, the type of photomultiplier used and its mode of operation, optical filtering, signal integration methods. The characteristics of the main readers are given. Four readers using original uncommon methods are described in greater details. It is planned to develop organic materials suitable for power laser optostimulation in the near infrared.

* Conférence présentée lors du Séminaire sur la dosimétrie in vivo en radiothérapie et en radiodiagnostic, Nice, 23-24 février 1989.

** Commissariat à l'énergie atomique, IPSN, Département de protection technique, DPT/ SIDR, BP 6, 92265 Fontenay-aux-Roses Cedex. 


\begin{abstract}
Thermoluminescence dosimetry is by far the most widely used technique for radiotherapy and will remain so for many years to come. The use of detector materials such as copper activated lithium borate (which is almost perfectly tissue equivalent) with new automated readers makes it possible to obtain high quality reproducible results quickly, with great advantages in monitoring doses given to patients.
\end{abstract}

\title{
1. INTRODUCTION
}

La dosimétrie par thermoluminescence (TL) $[8,11,14-15]$ s'est développée depuis une quinzaine d'années lorsque sont apparus sur le marché des lecteurs commerciaux fiables (1965). Les premiers lecteurs ont été des lecteurs manuels utilisés d'abord avec de la poudre puis plus tard avec des frittés. Par la suite, lorsque la surveillance dosimétrique opérationnelle s'est suffisamment développée, certains lecteurs ont été automatisés. II y a une douzaine d'années est arrivée la conception de lecteurs de badges dosimétriques TL entièrement automatiques. Depuis quelques années, sont apparus des lecteurs de poudre TL dont l'un d'eux a été spécialement étudié et développé par le CEA pour les applications hospitalières.

Notre but essentiel est d'apporter aux personnes concernées par ce type de dosimétrie et tout particulièrement aux physiciens d'hôpital, les informations nécessaires à une bonne connaissance de la technologie des lecteurs thermoluminescents afin d'adapter leur emploi et d'optimiser leur fonctionnement en dosimétrie hospitalière.

\section{DOSIMĖTRE THERMOLUMINESCENT}

Les détecteurs TL sont essentiellement constitués de composés minéraux ioniques cristallisés contenant des défauts ponctuels générés par adjonction d'un activateur. Les plus utilisés sont les suivants :

LiF : Dy

$\mathrm{CaSO}_{4}$ : Dy

$\mathrm{Li}_{2} \mathrm{~B}_{4} \mathrm{O}_{7}: \mathrm{Cu}$

$\mathrm{Li}_{2} \mathrm{~B}_{4} \mathrm{O}_{7}: \mathrm{Mn}$

$\mathrm{BeO}$ (non dopé)

$\mathrm{Al}_{2} \mathrm{O}_{3}$ (non dopée) dosimétrie individuelle, à la peau et d'extrémité, dosimétrie de l'environnement, dosimétrie en radiothérapie, dosimétrie en radiothérapie, dosimétrie individuelle et en radiothérapie, dosimétrie de criticité (insensible aux neutrons).

Les détecteurs se présentent sous deux formes possibles : poudres et frittés, excepté $\mathrm{BeO}$ toujours sous forme de fritté (à cause de sa toxicité chimique) et $\mathrm{Al}_{2} \mathrm{O}_{3}$ généralement en poudre.

\subsection{La poudre}

Le détecteur est généralement utilisé sous forme de poudre dans le cas où l'on désire obtenir la meilleure reproductibilité possible. Dans ce cas, la poudre homogénéisée présente, pour l'ensemble du lot, une même sensibilité. La réponse TL ne dépend que des 
conditions de lecture (conditions géométriques et thermiques) et en particulier de la masse de la poudre utilisée [6]. Suivant les produits, on peut effectuer une correction linéaire par rapport à la masse du détecteur afin de normaliser sa réponse à une valeur constante de la masse. On peut ainsi, en pesant chaque quantité de poudre à lire, obtenir une plus grande précision correspondant à un écart type sur la moyenne proche de $1 \%$ sur une dizaine de mesures.

En fonction de la nature et de l'énergie du rayonnement à mesurer, la poudre est conditionnée dans des sachets (rayonnement $X$ mou) ou dans des tubes à paroi plastique au-delà d'une centaine de keV. Lorsque la température est élevée, on utilise des conteneurs à paroi de verre ou métallique [5], c'est le cas de la dosimétrie des photons du cobalt ou du césium au contact des fûts dans les laboratoires de stockage des déchets de haute activité. La quantité, insluse dans chaque sachet ou tube, est fonction du nombre de mesures devant être effectuées par point d'irradiation. Très souvent, un prélèvement correspond à $20 \mathrm{mg}$ de poudre par mesure ; en général, 5 à 7 lectures sont effectuées par point de mesure $(150 \mathrm{mg})$. Ces valeurs sont couramment utilisées en radioprotection, toutefois l'utilisateur peut choisir la masse totale de poudre, la masse par prélèvement et la géométrie du conteneur en fonction de ses besoins propres.

\subsection{Le fritté}

L'utilisation en routine automatique de la poudre TL pose de nombreux problèmes inhérents à la nature même de la poudre (pulvérulence, débordement, versement, faible conduction thermique, etc...). Dans ce but, la poudre a été frittée en pastilles compactes d'environ $4.5 \mathrm{~mm}$ de diamètre et de $0,85 \mathrm{~mm}$ d'épaisseur pour l'étalon français et celui de Vinten. Les étalons étrangers, Harshaw, Panasonic, Alnor, etc. sont très voisins. En radioprotection, suivant les cas, les frittés sont utilisés seuls, regroupés par ensembles de 2 ou 4 afin d'obtenir plusieurs valeurs de la dose pour une meilleure statistique ou pour mesurer la dose sous différents écrans, $7-300-1000 \mathrm{mg} / \mathrm{cm}^{2}$ dans le cas des photons et $\left({ }^{6} \mathrm{Li}+{ }^{7} \mathrm{Li}\right)$ sous écran de cadmium dans le cas des neutrons [8]. En règle générale, on constate une dispersion de la sensibilité des frittés beaucoup plus grande que pour la poudre (un facteur de 2 à 3 à la fabrication). Les frittés trop sensibles présentent en général un "fading" plus important que la moyenne.

Toujours dans le cadre d'une utilisation de radioprotection et suivant les marques, les frittés peuvent être libres dans le tiroir du dosimètre (méthode CEA) ou fixés par pression à l'aide d'un film transparent résis" tant à la chaleur comme le capton. Cette dernière solution, quoique plus coûteuse, présente l'avantage d'une plus grande fiabilité ; on peut, dans ce cas, exercer une pression constante pour stabiliser l'impédance thermique de contact. 


\section{CONCEPTION D'UN LECTEUR DE THERMOLUMINESCENCE} $[7-8,14]$

\subsection{Généralités}

On dispose, à l'intérieur d'une chambre noire, un support chauffant au contact duquel se trouve le détecteur TL à mesurer. Au-dessus ou sur le côté, suivant la nature du matériau, un photomultiplicateur muni de filtres optiques appropriés détecte la lumière émise (fig. 1).

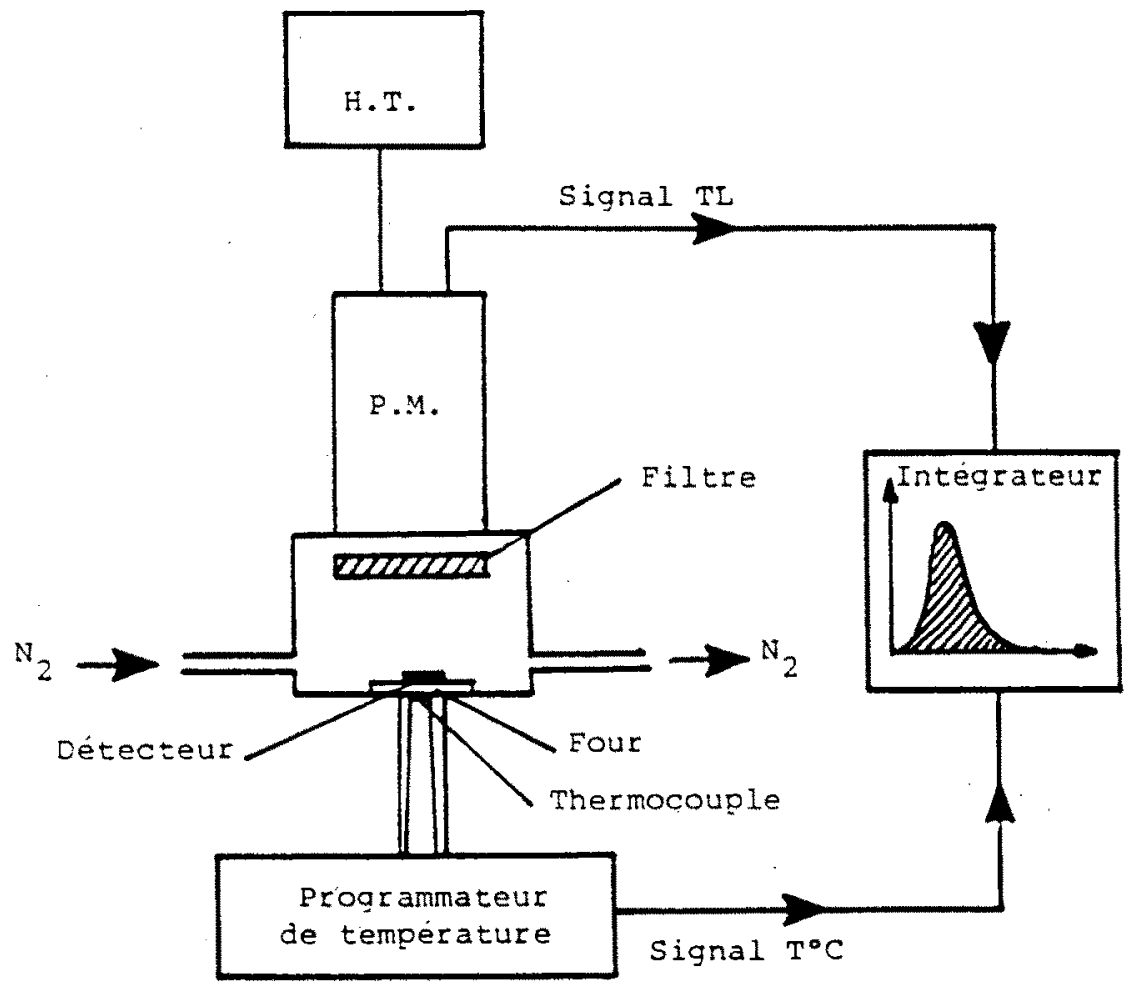

Fig. 1 - Schéma de principe d'un lecteur de TL.

\subsubsection{Cinétique de chauffage linéaire avec paller}

La quantité de lumière émise est fonction de la dose absorbée par le détecteur TL. Dans la majorité des cas, on recherche les conditions de lecture qui conduiront à une loi linéaire entre le signal et la dose, celui-ci étant, si possible, indépendant du passé du dosimètre : temps écoulé depuis l'irradiation, température de conservation, ambiance lumineuse, humidité, frictions mécaniques, etc... Dans ce but, ne seront lus que les pièges stables correspondant à des températures suffisamment élevées par rapport à la température ambiante. Depuis 20 ans, la majorité des lecteurs modernes utilisent la méthode du palier de préchauffage mis au point au CEA par PORTAL en 1965 (fig. 2). 


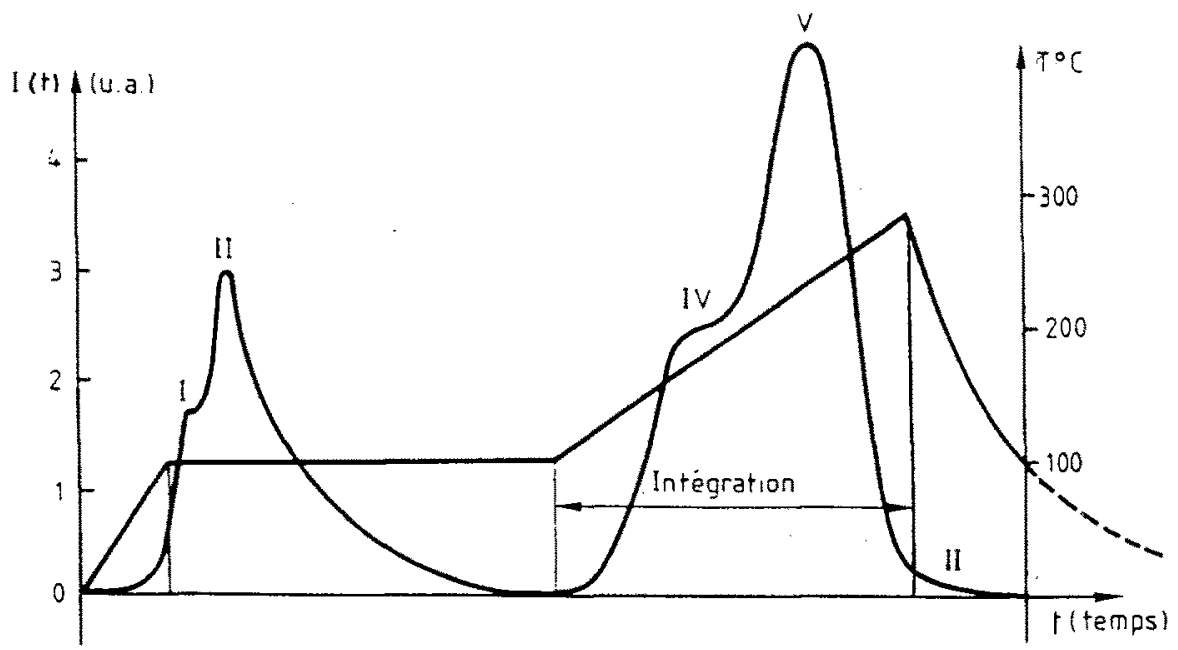

Fig. 2. - Cinbtique de chauffage linéaire avec paller.

\subsubsection{CInétique de chauffage non linéalre quast-Isotherme}

L'information utile est la quantité de lumière émise par le matériau TL, que cette dernière soit émise lentement ou rapidement. La grande majorité des lecteurs modernes utilisent un chauffage linéaire, mais certains lecteurs rapides, en particulier ceux développés au CEA, utilisent deux doigts chauffants isothermes, le premier pour le préchauffage, le second pour le chauffage (fig. 3).

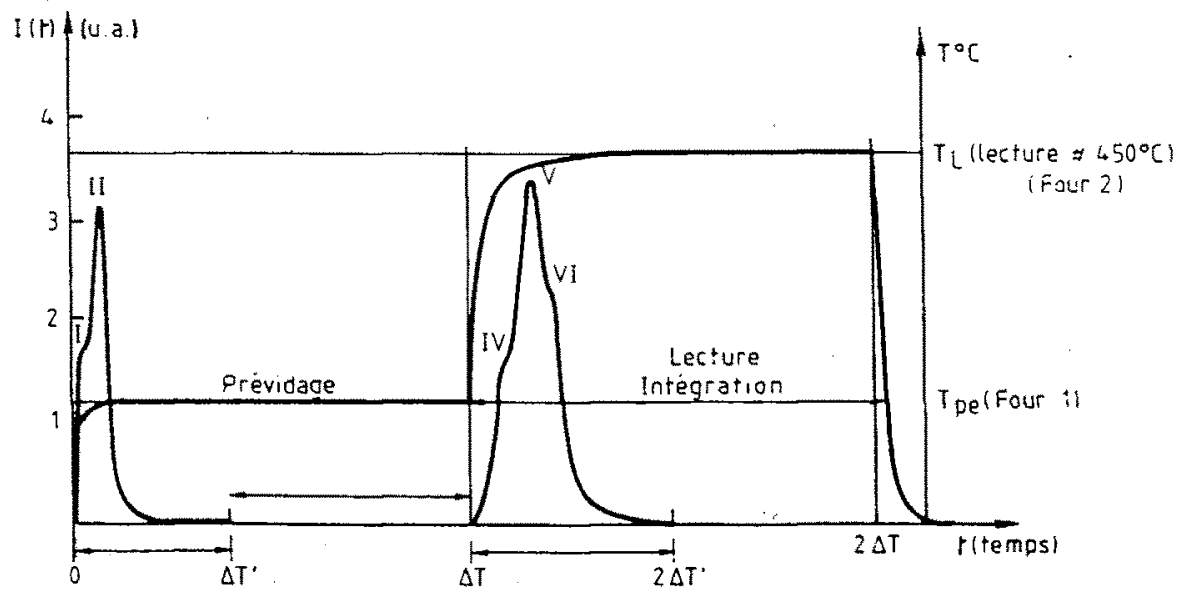

Fig. 3. - Cinótlque de chauffage non linéalre deux tempóratures. 


\subsubsection{Clrculation d'un gaz neutre}

Afin de diminuer la TL parasite due aux réactions photogènes générées par l'oxygène de l'air, particulièrement prépondérante aux doses faibles, la chambre noire est balayée par un courant d'azote ou d'argon dont le débit est un compromis entre la diminution du signal parasite, le refroidissement de la surface du détecteur et les effets d'entraînement aérodynamique, en particulier pour les poudres. Dans la plupart des cas, le débit est de 1 à $3 \mathrm{Imin}^{-1}$.

\subsection{Stimulation thermique de la thermoluminescence}

II est possible de stimuler la luminescence d'un détecteur TL de plusieurs façons ; la stimulation "optique", que l'on abordera en dernier lieu, n'est pas une thermoluminescence vraie.

\subsubsection{Chauffage par conduction thermique}

Le détecteur est chauffé par contact thermique. Dans ce cas, l'interface entre l'élément chauffant et le détecteur joue un rôle primordial sur la vitesse de chauffage et sur l'écart de statisme thermique. De nombreux fonctionnements défectueux proviennent d'un défaut de contact dû à la poudre intercalaire, aux états de surface, à l'oxydation, etc.

\subsubsection{Chauffage par convection thermique}

Pour éviter les problèmes éventuels dus à de mauvais contacts thermiques, il est possible de chauffer le fritté, mais non la poudre, par projection d'azote chaud $\left(400\right.$ à $\left.500{ }^{\circ} \mathrm{C}\right)$. L'asservissement thermique du détecteur est très difficile à réaliser et la cinétique thermique est imprécise, par contre, on obtient généralement moins de mesures anormales.

\subsubsection{Chauffage par rayonnement}

II est également possible de chauffer rapidement le détecteur par rayonnement infra-rouge (IR). On a alors le choix entre deux types de sources de lumière:

1. Une source à filament incandescent qui émet un spectre de lumière allant du proche UV à I'IR moyen. On utilise fréquemment une lampe à halogène munie d'un miroir réflecteur et concentrateur sur lequel est déposé, par métallisation sous vide, un dépôt d'or. Ce dernier a pour rôle d'optimiser la réflexion des photons IR. Avec une lampe de $150 \mathrm{~W}$ on obtient couramment des vitesses de chauffage de $10^{\circ} \mathrm{C} / \mathrm{s}$ et des températures atteignant $500{ }^{\circ} \mathrm{C}$ [7-8].

2. Le chauffage par laser $\mathrm{CO}_{2}$ (longueur d'onde : 10,6 $\mu \mathrm{m}$ ) est également utilisé. II s'agit, en général, d'un laser fonctionnant en semi-continu (pompage électromagnétique hyperfréquence) d'une puissance lumineuse variant de 1 à $100 \mathrm{~W}$. La vitesse de chauffage et la température atteinte sont fonction de la puissance et de la durée de la stimulation. 
Avec un laser de $20 \mathrm{~W}$ la durée de la mesure s'effectue en 2 à $3 \mathrm{~s}$. La limitation de la fréquence des lectures est essentiellement due aux contraintes mécaniques de transfert des dosimètres. Avec des lasers de puissance, la vitesse de chauffage peut atteindre $10000^{\circ} \mathrm{C} / \mathrm{s}$. La ponctualité du faisceau laser peut être mise à profit pour répéter les mesures sur un même échantillon en diverspoints de sa surface. En effet, quand la vitesse de chauffage est très grande et la durée très brève, il y a une faible diffusion thermique, en particulier pour les détecteurs de faible épaisseur $<0,1 \mathrm{~mm}$. Cette technique a été développée pour limagerie par thermoluminescence et pour la cartographie des doses [1].

\subsubsection{Stimulation optique}

Pour réaliser la stimulation optique, on utilise un laser à impulsion de très forte puissance dans le proche IR (par exemple $\lambda=1,06 \mu \mathrm{m}$ ). La puissance crête est de quelques dizaines de $M W$ et la durée de l'impulsion de 10 à $50 \mathrm{~ns}$. Compte-tenu de l'énorme intensité lumineuse, les phénomènes d'excitation multiphotonique sont très fréquents et on peut ainsi, pratiquement sans chauffer, générer l'émission de la "photo" plutôt que de la "thermo" luminescence. II est ainsi envisageable d'utiliser des détecteurs organiques équivalents au tissu. II faut toutefois noter que les produits thermoluminescents ne sont pas nécessairement opto-stimulables.

\subsection{Détection optique}

Etant donné les faibles intensités lumineuses émises par les produits $T L$, en particulier pour les doses faibles, courantes en radioprotection, le photomultiplicateur (PM) est universellement employé. Toutefois, les progrès accomplis en électronique linéaire permettent d'envisager pour les doses élevées ( $>1 \mathrm{~Gy}$ ), un capteur optique tel que l'association d'une cellule photoélectrique et d'un amplificateur à courant faible. On peut également envisager l'emploi des cristaux ou des mosaïques CCD refroidies (actuellement utilisées dans les camescopes). En effet, certains télescopes optiques (Meudon) utilisent avec succès ces mosaïques en intégration pendant plusieurs jours à la place des pellicules photographiques. Cette technique est couramment utilisée en chronofluorimétrie dans les analyseurs multicanaux optiques.

\subsubsection{Photomultipllcateur}

Un PM se comporte comme un générateur de courant. La tension obtenue sur l'anode est, dans une grande étendue, proportionnelle à l'impédance de la charge utilisée.

- Gain et linéarité. Le gain du PM dépend du nombre de dynodes multiplicatrices et de la tension interdynode. Pratiquement, il peut varier de $10^{4}$ à $10^{8}$. Utilisée en régime continu, la linéarité est sensiblement parfaite du bruit de fond jusqu'à une valeur proche de celle du courant du pont de polarisation. En régime impulsionnel, elle peut être très élevée et 
n'est limitée que par la dépolarisation des dynodes et l'effet de charge d'espace au niveau des derniers étages.

- Réponse spectrale. Suivant l'énergie du photon incident, la photocathode a une probabilité différente d'émettre un photo-électron. Ainsi le choix d'un PM est fonction de l'adéquation entre sa réponse spectrale et le spectre optique du signal lumineux à détecter. Dans la majorité des produits $T L$, excepté $\mathrm{Li}_{2} \mathrm{~B}_{4} \mathrm{O}_{7}: \mathrm{Mn}$, Si qui émet dans le rouge, les PM sensibles dans le bleu et le proche ultra-violet seront aptes a la détection TL de routine. Les réponses spectrales du type $\mathrm{S} 11$ ou $\mathrm{S} 13$ seront les plus fréquemment employées ; en outre, elles sont peu sensibles dans le rouge et I'IR, correspondant au rayonnement du corps noir émis par une surface chaude.

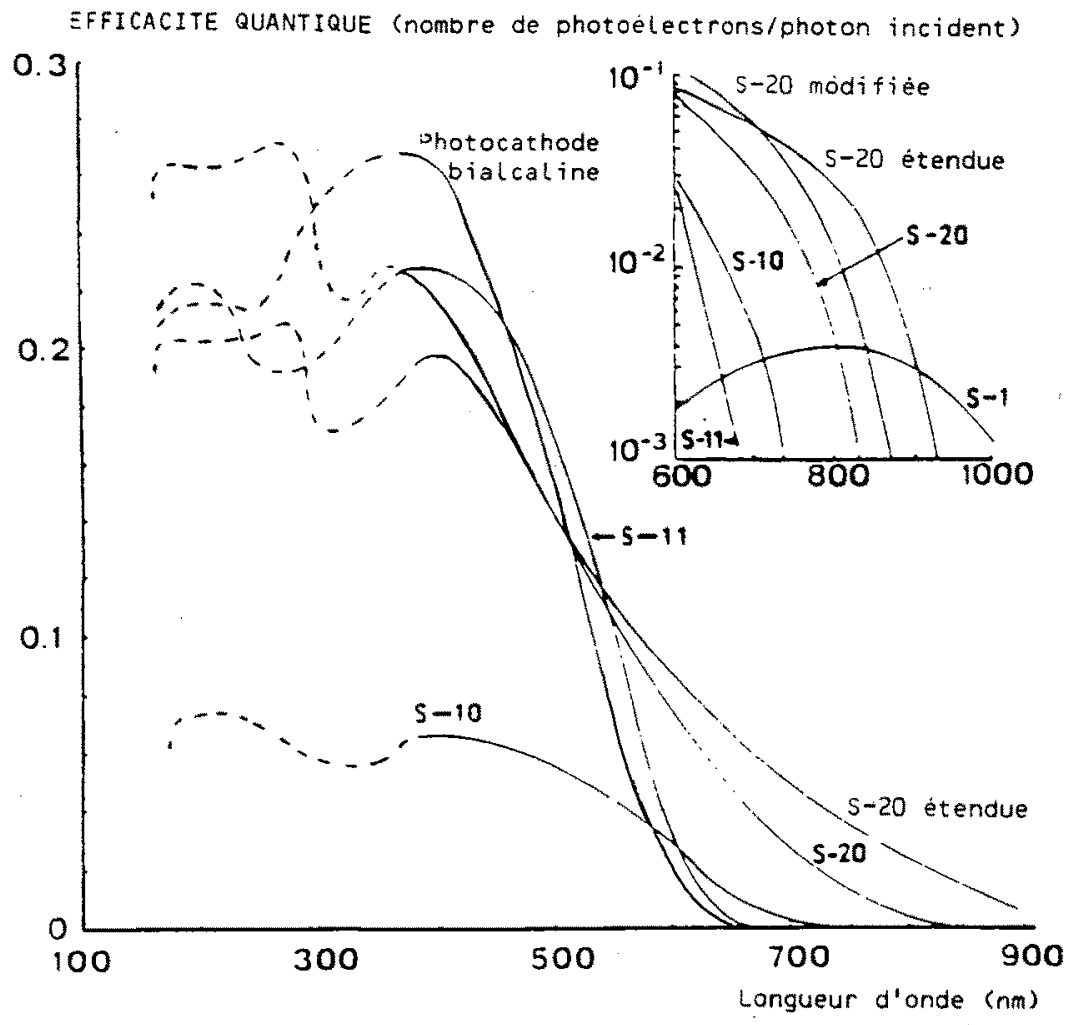

Fig. 4. - Réponses spectrales de quelques types de photocathodes.

- Bruit de fond. Le bruit de fond d'un PM dépend de la qualité de la photocathode, de celle des isolants (verre, silice...), de la tension de polarisation et de la température. Le bruit de fond ou courant d'obscurité évolue avec la température selon une loi de Boltzmann. A basse température, ce dernier sera très faible (variation d'un facteur 2 par écart 
de $40^{\circ} \mathrm{C}$ au voisinage de $0^{\circ} \mathrm{C}$ ). Notons que la sensibilité de la photocathode baisse également avec la température mais de façon bien moins importante. Aux températures inférieures à $5^{\circ} \mathrm{C}$, il faut faire attention aux condensations d'eau qui peuvent opaliser la photocathode et créer des courants de fuite instables et importants.

Une composante transitoire très importante du bruit de fond apparaît à la mise sous tension et décroît au cours du temps (quelques minutes à plusieurs jours suivant les cas). Ce courant d'obscurité correspond à l'ionisation par le champ électrique appliqué des molécules de gaz résiduel qui, une fois ionisées, seront piégées par les éléments métalliques sous tension tels que les surfaces "getter". Le courant d'obscurité diminue ainsi jusqu'à tendre vers une valeur minimale.

Une fois sous tension, un PM ne doit jamais être arrêté si ce n'est pour des raisons de sécurité.

\subsubsection{Types de fonctionnement}

Suivant les caractéristiques de la résistance de charge anodique, le PM pourra travailler en continu ou en régime impulsionnel.

- Fonctionnement en continu. Dans ce premier cas, la constante de témps du circuit d'anode est grande par rapport à la durée séparant l'émission de photoélectrons consécutifs. II y aura intégration exponentielle et établissement d'un régime d'équilibre quasi-continu. Avec ce type de fonctionnement, on ne peut faire aucune distinction sur l'origine des courants recueillis, courant dû au signal lumineux ou courant dû au bruit de fond des dynodes.

- Fonctionnement en régime impulsionnel. Dans ce deuxième cas, la constante du circuit d'anode est courte par rapport à la durée séparant l'émission de 2 photoélectrons consécutifs. Si le gain du PM est assez élevé, on obtient alors une impulsion électrique par photoélectron ou paquet de photoélectrons incidents. Les électrons, thermiquement générés par les électrodes autres que la photocathode, seront moins multipliés et donneront des impulsions plus petites. Ainsi, en discriminant leur amplitude, il sera possible de s'affranchir d'une grande partie du bruit thermique, excepté de celui de la photocathode.

Le lecteur TL mis au point sur ce principe, il y a plus de 15 ans, par Valladas-Brou-Portal, permet (avec les filtres optiques appropriés) de mesurer les produits TL jusqu'à $1000{ }^{\circ} \mathrm{C}$.

\subsubsection{Flitre optique}

Le rôle du filtre optique est d'éliminer de la TL à mesurer les photons générés par l'émission du corps noir à la température de lecture. Ce seront donc des filtres passe-bas en longueur d'onde. Le choix de la valeur de coupure est un compromis fonction du rapport signal/bruit 
du spectre optique du produit TL et de la sensibilité que l'on désire obtenir. La figure 5 représente le spectre de transmission de quelques filtres couramment utilisés.
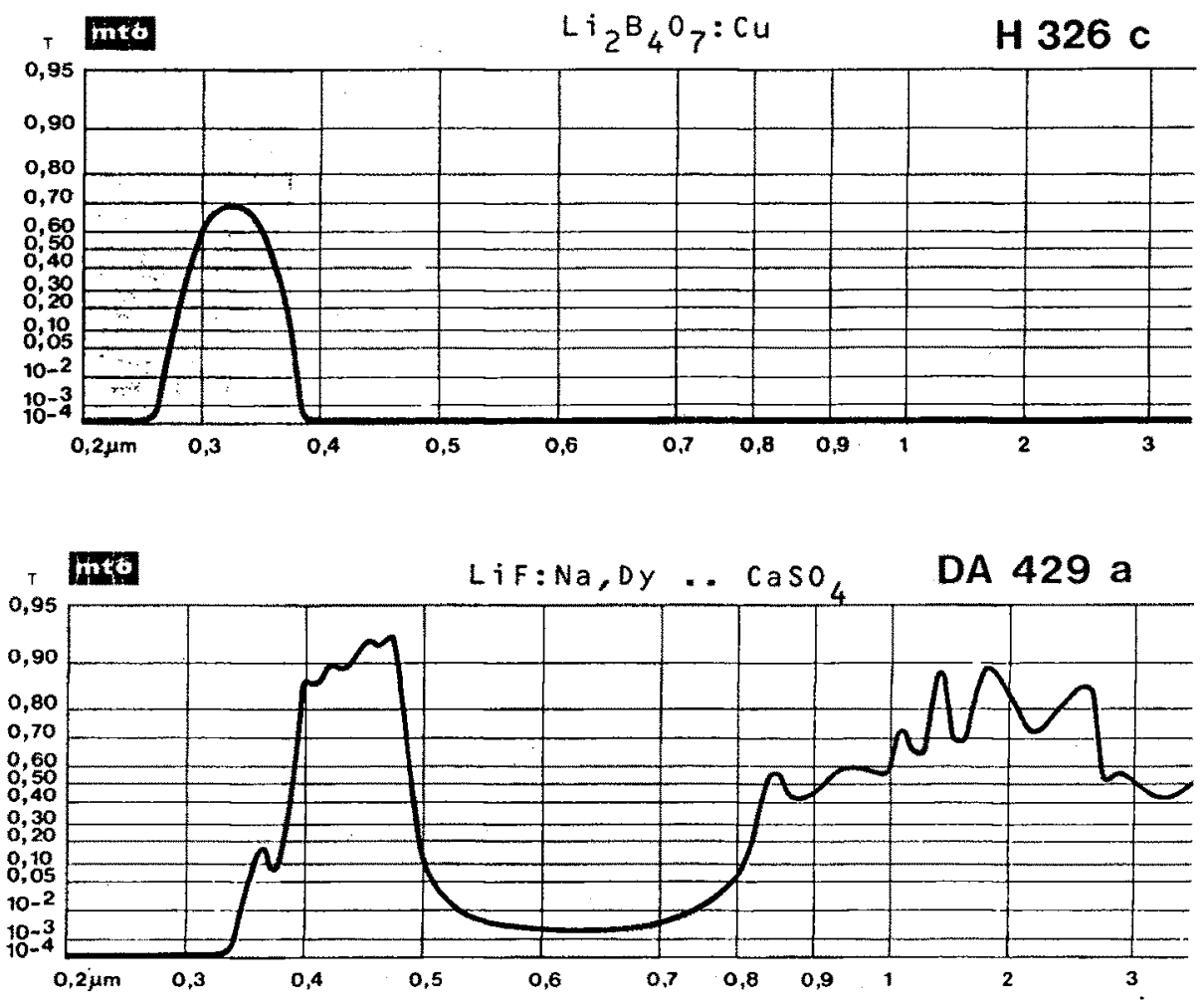

Fig. 5. - Spectres de quelques filtres utilisés [13].

\subsection{Intégration du signal de thermoluminescence}

On constate que l'allure du thermogramme [Signal $\left.=f\left(D o s e, T^{\circ} \mathrm{C}\right)\right]$ dépend fortement des conditions de la stimuiation thermique. Or, le nombre de pièges ayant capturé un électron sous l'effet de l'irradiation est fini. II suffira donc, pour avoir une mesure réelle de la dose, d'utiliser un paramètre proportionnel au nombre de pièges chargés. Pour cela, on intègre le signal TL entre deux températures choisies en fonction des caractéristiques du produit utilisé, de l'allure du thermogramme, du bruit de fond et éventuellement de la dose. Il existe trois techniques possibles pour intégrer le signal TL. 


\subsubsection{Intégration analogique capacitative (fig. 1$)$}

Cette méthode est la plus ancienne, la plus problématique et maintenant la moins utilisée sur les lecteurs conçus de nos jours. Le courant fourni par le PM charge un condensateur réel ou fictif (effet Miller). La tension à ses bornes s'écrit :

$$
V(t)=\frac{1}{C} \int_{t_{1}}^{t_{2}} i(t) d t \quad \text { avec } i(t) \text { proportionnel au flux TL. }
$$

Un circuit de remise à zéro (relais ou transistor à effet de champ) vide le condensateur ; celui-ci est alors apte à intégrer le courant d'une nouvelle mesure. Dans ces lecteurs, la valeur analogique de la tension est affichée sur un voltmètre à cadre ou convertie en un nombre à l'aide d'un voltmètre numérique. La grande impédance statique de ce type d'intégrateur le rend très sensible aux courants parasites générés par les variations de température et l'humidité ambiante.

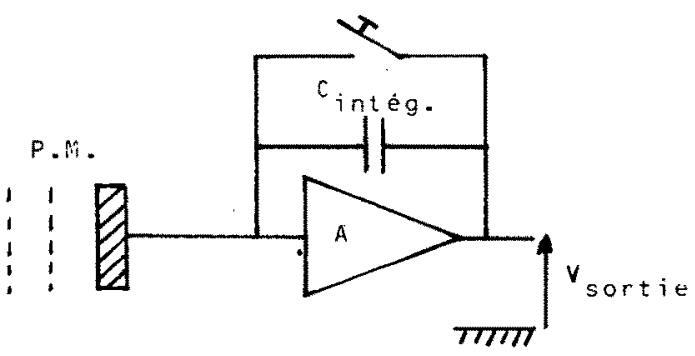

Fig. 6. - Intégration capacitive.

\subsubsection{Intégration numérique par convertisseur courant-fréquence (fig. 7)}

Le courant fourni par le PM est converti en tension à l'aide d'un préamplificateur convertisseur courant-tension. La tension en sortie de l'amplificateur s'écrit : $V=-R i$ où $R$ est la résistance de contre réaction souvent en parallèle avec une capacité de filtrage. Cette tension (sous faible impédance) est transformée en un signal carré de fréquence proportionnelle à l'aide d'un convertisseur tension-fréquence : par exemple, chargement d'un circuit capacitif à seuil déclenché par un transistor unijonction, un amplificateur à contre réaction, ou encore un circuit monostable autodéclenché dont la tension de cycle est piloté par le signal à convertir. Actuellement, on dispose, sur le marché, de circuits de conversion tension-fréquence intégrés de très bonne qualité avec des offsets inférieurs à $0,1 \mathrm{mV}$ correspondant à une fréquence de base de $1 \mathrm{~Hz}$ pour une dynamique de $10^{5}$ à $10^{7}$.

Notons qu'un convertisseur n'est jamais linéaire aux faibles tensions et la dynamique maximale utile ne dépasse pas $10^{5}$ en moyenne. Un même convertisseur ne pourra pas servir tel quel de $10 \mu \mathrm{Gy}$ à $100 \mathrm{~Gy}$, 
c'est-à-dire pour deux applications simultanées de radioprotection et de radiophysique. II faudra réduire la dynamique par un changement du gain du préamplificateur ou par la modification (absorption optique) de l'intensité de la fluorescence atteignant le PM.

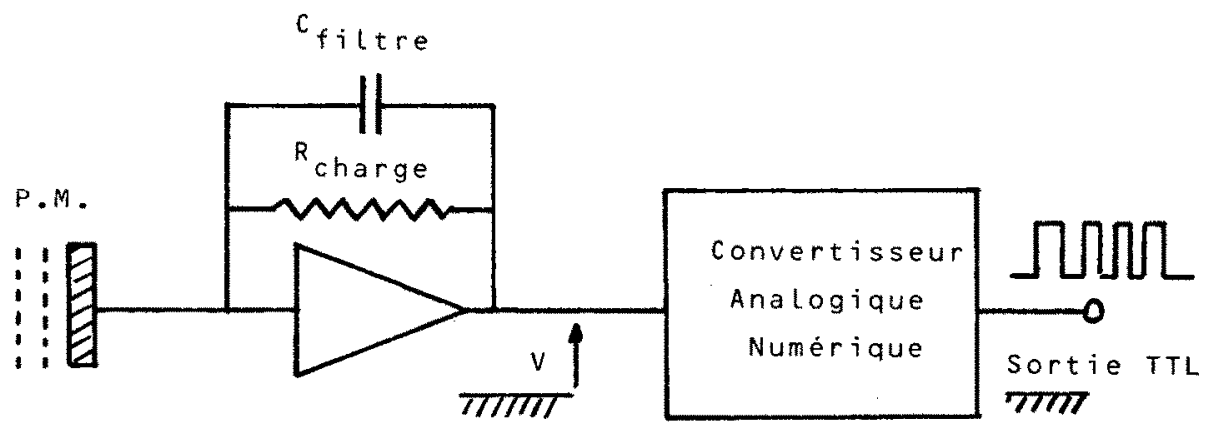

Fig. 7. - Intégration numérique par conversion tension-fréquence.

\subsubsection{Intégration par comptage de photons (fig. 8)}

Dans ce cas, l'intégration du flux lumineux TL consiste à compter les impulsions d'anode du PM correspondant aux photoélectrons ou aux paquets de photoélectrons émis par la photocathode. Une simple échelle de comptage sera suffisante. II faut, toutefois, noter que la linéarité de la réponse entre le flux lumineux et le comptage effectif est liée à l'intensité de la lumière. La linéarité ne sera observée que pour les faibles intensités. Pour les fortes valeurs, il y aura un effet progressif d'empilement entraînant une non linéarité avec le flux optique incident.

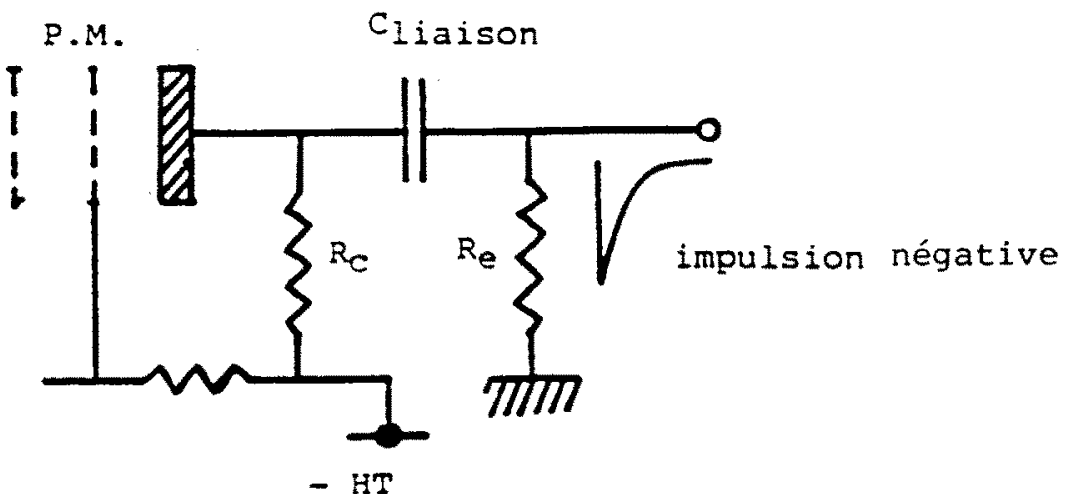

Fig. 8. - Intégration numérique par comptage de photons. 
La distribution en amplitude des impulsions fournies par ce circuit (fig. 9) a l'allure d'une fonction exponentielle décroissante ; on montre que la probabilité relative d'obtenir une impulsion d'amplitude donnée ne dépend que des caractéristiques de la photocathode, de sa sensibilité spectrale et de son rendement quantique.

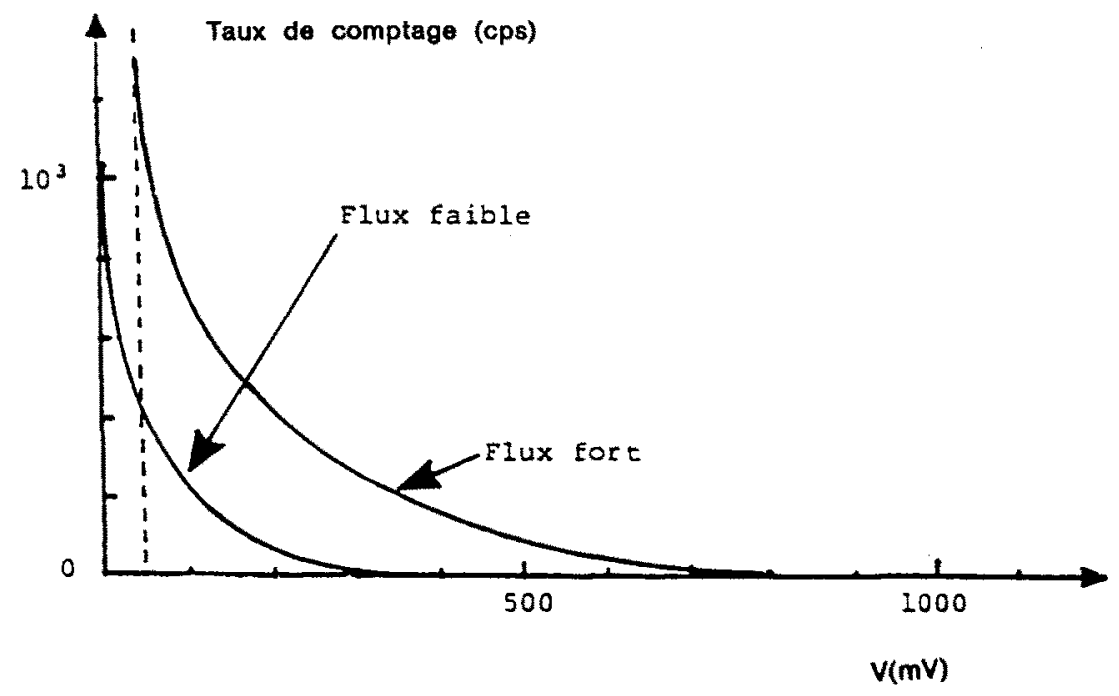

Fig. 9. - Spectre en amplltude des impulsions fournles par un PM.

La dynamique en fréquence d'un tel système dépend de la constante de temps du circuit d'anode du PM. Avec un circuit à grande fréquence de coupure haute, on atteint un taux de comptage de $10^{7} \mathrm{~Hz}$. Ceci est à comparer avec la dynamique classique des convertisseurs à circuits intégrés, $10^{5}$ en moyenne.

\section{LECTEURS COMMERCIAUX (12) (Tableaux II et III)}

Aux lecteurs non commercialisés (tableau II) ou actuellement disponibles sur le marché (tableau III), il faut rajouter le lecteur français CEA (PCL) spécialement conçu pour les physiciens d'hôpital et également utilisable en radioprotection. 
TABLEAU \|

Caractéristiques de quelques lecteurs semi-commerciaux

\begin{tabular}{|c|c|c|c|c|c|c|}
\hline Institution & Pays & Modèle & $\begin{array}{l}\text { Système de } \\
\text { chauffage }\end{array}$ & $\begin{array}{l}\text { Type du } \\
\text { défecteut }\end{array}$ & $\begin{array}{c}\text { Manuel } \\
\text { Automatique }\end{array}$ & Remarques \\
\hline AECL, Chaik River & Canada & - & Ruban chauffant & $\begin{array}{l}\text { Cartes dos. indiv. } \\
\text { Disques de LiF sur } \\
\text { un ruban de capton }\end{array}$ & Automalique & $\begin{array}{l}\text { Controle } \\
\text { par } \\
\text { microprocesseur }\end{array}$ \\
\hline $\begin{array}{l}\text { Bureau of Radiation } \\
\text { and Medical Devices, } \\
\text { Ottawa }\end{array}$ & Canada & - & Ruban chauffant & $\begin{array}{l}\text { Cartes dos. indiv. } \\
\text { Disques de LiF sur } \\
\text { un ruban de capton }\end{array}$ & Automatique & - \\
\hline $\begin{array}{l}\text { Riso National } \\
\text { Laboratory, Roskilde }\end{array}$ & Danemark & - & Azote chaud & $\begin{array}{l}\text { Dosimètres indiv. } \\
\text { frittés de LiF et } \\
\left.\mathrm{Li}_{2} \mathrm{~B}_{4} \mathrm{O}_{7}\right)\end{array}$ & Automatique & - \\
\hline $\begin{array}{l}\text { CEA, } \\
\text { Fontenay-aux-Roses }\end{array}$ & France & - & Doigt chautfant & $\begin{array}{l}\text { Dosimètres indu } \\
\text { (Frittés de LiF) }\end{array}$ & Automatique & - \\
\hline $\begin{array}{l}\text { Central Res. Inst. for } \\
\text { Physics, Budapest }\end{array}$ & Hongrie & NHZ 203/204 & Planchette & $\begin{array}{l}\text { Détecleurs solides } \\
\text { libres et poudres }\end{array}$ & Manuel & - \\
\hline $\begin{array}{l}\text { Central Res, Inst, for } \\
\text { Physics, Budapest }\end{array}$ & Hongrie & NA-206E & Filament chauffant & $\begin{array}{l}\mathrm{CaSO}_{4} \mathrm{Dy}(\mathrm{Tm}) \text { en } \\
\text { ampoule }\end{array}$ & Manuel & - \\
\hline WNo, Anthem & Pays-Bas & Système & Azote chaud & $\begin{array}{l}\text { Dosimètres indiv. } \\
\text { (tout détecteut } \\
\text { solide) }\end{array}$ & Alstomatique & - \\
\hline TNO, Arnhem & Pays-6as & Lecteut & Azote chaud & $\begin{array}{l}\text { Détecteurs solides } \\
\text { libres }\end{array}$ & Automatique & - \\
\hline $\begin{array}{l}\text { Inst. Nuclear Physics, } \\
\text { Krakow }\end{array}$ & Pologne & ASTL-810 & Bande chauffante & $\begin{array}{l}\text { Cartes dos. indiv. } \\
\text { (Disque de Lif) }\end{array}$ & Automatique & - \\
\hline NRPB, Harwell & Grande-Bretagne & - & Doigt chauffant & $\begin{array}{l}\text { Plaques de dos. } \\
\text { pers. (Lif dans un } \\
\text { disque en téflon) }\end{array}$ & Automatique & - . \\
\hline EML, New York & USA & - & Planchette & $\begin{array}{l}\text { Détecteurs solides } \\
\text { fibres et poudres }\end{array}$ & Manuel & - \\
\hline $\begin{array}{l}\text { International Sensor } \\
\text { Technology, Pullman, } \\
\text { WA }\end{array}$ & USA & - & Laser & $\begin{array}{l}\text { Détecteurs spéciaux } \\
\text { dans des supports } \\
\text { spéciaux }\end{array}$ & Automatique & - \\
\hline $\begin{array}{l}\text { Josef Stelan Inst., } \\
\text { Ljubejana }\end{array}$ & Yougoslavie & ins MR-200 & Planchette (?) & Detecteurs solides & Automalique & - \\
\hline $\begin{array}{l}\text { Josef Stefan Inst. } \\
\text { Ljubejana }\end{array}$ & Yougosiavie. & TDS-08 & (?) & $\begin{array}{l}\text { Dos. TLD.08 avec } \\
\text { un fritte de } \\
\text { Cafi : Mn }\end{array}$ & $\begin{array}{l}\text { Semi* } \\
\text { Automatique }\end{array}$ & - \\
\hline
\end{tabular}


TABLEAU $\| I$

Caractéristlques de quelques lecteurs commerclaux

\begin{tabular}{|c|c|c|c|c|c|c|}
\hline Institution & Pays & Modele & $\begin{array}{l}\text { Système de } \\
\text { chauffage }\end{array}$ & $\begin{array}{l}\text { Type du } \\
\text { détecteur }\end{array}$ & $\begin{array}{c}\text { Manuel } \\
\text { Automatique }\end{array}$ & Remarques \\
\hline Altor & Finlante & Dosacus & Bande chauffante & $\begin{array}{l}\text { Dos. indiv. Gusqu'à } \\
4 \text { détecteurs) }\end{array}$ & Automatique & $\begin{array}{l}\text { Controlé par } \\
\text { microprocesseur } \\
\text { complage des photons }\end{array}$ \\
\hline Kyokiko & Japon & 2500 & Planchette & $\begin{array}{l}\text { Détecteurs solides } \\
\text { libres (disques frittes, } \\
\text { poudre encapsule } \\
\text { sous yerre) }\end{array}$ & Manuel & $\begin{array}{l}\text { Controlé par } \\
\text { microprocesseur }\end{array}$ \\
\hline $\begin{array}{l}\text { Nationall } \\
\text { Panasonic }\end{array}$ & Japon & $\begin{array}{l}\text { UD-702E } \\
t \\
\text { UD-710 }\end{array}$ & $\mathbb{R}$ & $\begin{array}{l}\text { Dos. indiv. (matériaux } \\
\text { en poudre dans I film } \\
\text { de silicone, jusqu'à } \\
4 \text { détecteurs) }\end{array}$ & $\begin{array}{l}\text { Manuel } \\
\text { Automalique }\end{array}$ & $\begin{array}{l}\text { Contrôlé par } \\
\text { microprocesseur } \\
\text { comptage des photons } \\
\text { aux aibles doses }\end{array}$ \\
\hline $\begin{array}{l}\text { Nalionail } \\
\text { Panasonic }\end{array}$ & Japon & UD-512A & Alr chaud & $\begin{array}{l}\text { Frittés libres et } \\
\text { poudrc encapsulee } \\
\text { sous verre }\end{array}$ & Manuel & $\begin{array}{l}\text { Controlé par } \\
\text { microprocesseur }\end{array}$ \\
\hline Vinten & Royaume-Uni & 654-Toledo & $\begin{array}{l}\text { Plateaul } \\
\text { Lamelle }\end{array}$ & $\begin{array}{l}\text { Détecteurs libres } \\
\text { et poudre }\end{array}$ & Manuel & $\begin{array}{l}\text { Chargeur externe } \\
\text { de } 30 \text { échantillons }\end{array}$ \\
\hline Vinten & Royaume-Uni & $\begin{array}{l}\text { 654E-Unisal } \\
\text { Toledo }\end{array}$ & Plateaullamelle & $\begin{array}{l}\text { Cartes dos. indiv, ou } \\
\text { dos. dextremite code } \\
\text { sur ruban }\end{array}$ & Manuel & $\begin{array}{l}\text { Conirôlé par } \\
\text { microprocessseut }\end{array}$ \\
\hline Vinten & Royaume-Uni & $654 \times$ Toledo & Plateaullamelle & $\begin{array}{l}\text { Dos, ruban } \\
\text { d'extrémité }\end{array}$ & Automatigue & $\begin{array}{l}\text { Conitólé par } \\
\text { microprocesseur }\end{array}$ \\
\hline Vinten & Royaume-Uni & $\begin{array}{l}690 \text { mini } \\
\text { Toledo }\end{array}$ & Piateaullamelle & Cartes dos. indiv, & Manuel & $\begin{array}{l}\text { Contrólé par } \\
\text { microprocesseur }\end{array}$ \\
\hline Vinten & Royaume-Uni & $\begin{array}{l}813 \text { Auto: } \\
\text { matique }\end{array}$ & Plateaullamelle & Cartes dos indiv. & Automatique & $\begin{array}{l}\text { Contrôlé par } \\
\text { microprocesseur }\end{array}$ \\
\hline Harshaw & USA & $2000 \mathrm{~A} / \mathrm{B}$ & Planchette & $\begin{array}{l}\text { Détecteurs solides } \\
\text { libres, poudre }\end{array}$ & Manuel & $\begin{array}{l}\text { Systeme composé de } \\
\text { deux unitess separées }\end{array}$ \\
\hline Harshaw & USA & $2000 \mathrm{C}$ & $\begin{array}{l}\text { Doigt chauffant } \\
\text { Planchette }\end{array}$ & $\begin{array}{l}\text { Cartes dos, indiv. } \\
\text { détecteurs solides } \\
\text { et poudies }\end{array}$ & Manuel & $\begin{array}{l}\text { Doit être utilisè avec te } \\
\text { pico-ampèremètre } \\
2000 \mathrm{~B}\end{array}$ \\
\hline Harshaw & Usta & 2271 & $\begin{array}{l}\text { Doigt chauffant } \\
\text { Pianchette }\end{array}$ & $\begin{array}{l}\text { Cartes dos. indliv. } \\
\text { détecteurs et poudres }\end{array}$ & Automatique & $\begin{array}{l}\text { Doil être utitisé avec le } \\
\text { pico-ampèremètre } \\
20008\end{array}$ \\
\hline Hanshaw & USA & $2000 \mathrm{P}$ & $\begin{array}{l}\text { Monté avec un } \\
\text { filament chauffant }\end{array}$ & Dos. sous ampoule & Manuel & $\begin{array}{l}\text { Doif être utilisé avec le } \\
\text { pico-ampèremètre } \\
2000 \mathrm{~B}\end{array}$ \\
\hline Harshaw & USA & $\begin{array}{l}20000 \\
\text { (attas) }\end{array}$ & Bande chauffante & Disquas & & $\begin{array}{l}\text { Dolt être utilisé avec le } \\
\text { pico-ampèremètre } \\
2000 \mathrm{~B}\end{array}$ \\
\hline Harshaw & USA & $3000(A)$ & Planchelte & $\begin{array}{l}\text { Détecteurs solides, } \\
\text { Wibres, poudres }\end{array}$ & Manuel & $\begin{array}{l}\text { Similaire au } 2000 \mathrm{~A} / \mathrm{B} \\
\text { mais en une setile unité }\end{array}$ \\
\hline Harshaw & USA & $4000 \mathrm{AB}$ & $\begin{array}{l}\text { Planchette } \\
\text { Doigt chauffant }\end{array}$ & $\begin{array}{l}\text { Détecteurs libres } \\
\text { Cartes dos. indiv. }\end{array}$ & Manuel & $\begin{array}{l}\text { Contrölé par } \\
\text { microprocesseur } \\
\text { Sélection des para* } \\
\text { mètres grấce á un menu }\end{array}$ \\
\hline Harshaw & USA & $8000(C)$ & Doigt chauffant & Cartes dos, indiy. & Automatique & $\begin{array}{l}\text { Sélection des para- } \\
\text { mètres grâce a un menu }\end{array}$ \\
\hline $\begin{array}{l}\text { Teledyne } \\
\text { Isotopes }\end{array}$ & USA & 7300 & Planchette & $\begin{array}{l}\text { Détecteurs libres, } \\
\text { poudres }\end{array}$ & Manuel & \\
\hline $\begin{array}{l}\text { Teledyne } \\
\text { lsotopes }\end{array}$ & USA & 8300 & Doigt chauffant & Dos. indiv. (PadiGuard) & Manuel & \\
\hline $\begin{array}{l}\text { Teledyne } \\
\text { lsotopes }\end{array}$ & USA & 9150 & Doigt chauffant & Dos. indiv. (RadiGuard) & Automatique & $\begin{array}{l}\text { Contrôlé par } \\
\text { microprocesseur }\end{array}$ \\
\hline Victoreen & USA & 2800 & $\begin{array}{l}\text { Planchette } \\
\text { réceptacle en } \\
\text { forme de bulle }\end{array}$ & $\begin{array}{l}\text { Détecteurs solides, } \\
\text { poudres, ampoules }\end{array}$ & Manuel & $\begin{array}{l}\text { Cycle de chauffage } \\
\text { programmable }\end{array}$ \\
\hline Victoreen & USA & 2810 & $\begin{array}{l}\text { Planchette } \\
\text { réceptacle en } \\
\text { forme de bulle }\end{array}$ & $\begin{array}{l}\text { Détecteurs solides, } \\
\text { poudres, ampoules }\end{array}$ & Manuel & $\begin{array}{l}\text { Cycle de chauffage } \\
\text { programmable }\end{array}$ \\
\hline
\end{tabular}




\section{DESCRIPTION DE QUELQUES LECTEURS PARTICULIERS}

Parmi les différents lecteurs présentés, nous allons examiner de façon plus détaillée 4 lecteurs fonctionnant selon des modes de chauffages différents et n'utilisant pas de lame ou de planchette chauffante comme le LDT de Saphymo, les Harshaw 3000 et 4000, le Victoreen 2800 ou le Vinten Toledo ou Minitoledo, à savoir :

- Panasonic UD-710 (Japon) à chauffage optique,

- TNO (Pays-Bas) à chauffage par gaz chaud,

- IST (USA) à chauffage laser,

- PCL (CEA France) à doigts chauffants isothermes.

\subsection{Panasonic à chauffage optique infra-rouge [7]}

II s'agit d'un lecteur automatique de frittés dont les diagrammes des figures 10 et 11 précisent le fonctionnement.

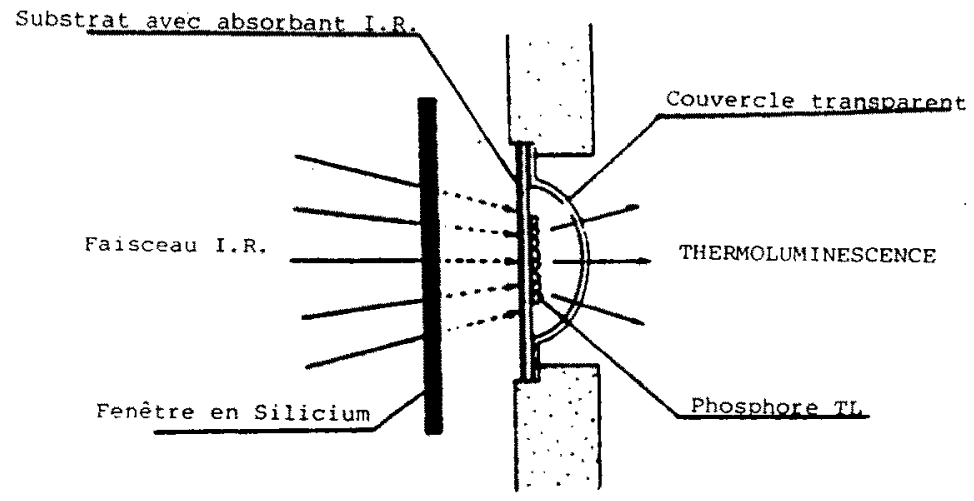

Fig. 10. - Panasonic a chauffage IR. Principe de mesure.

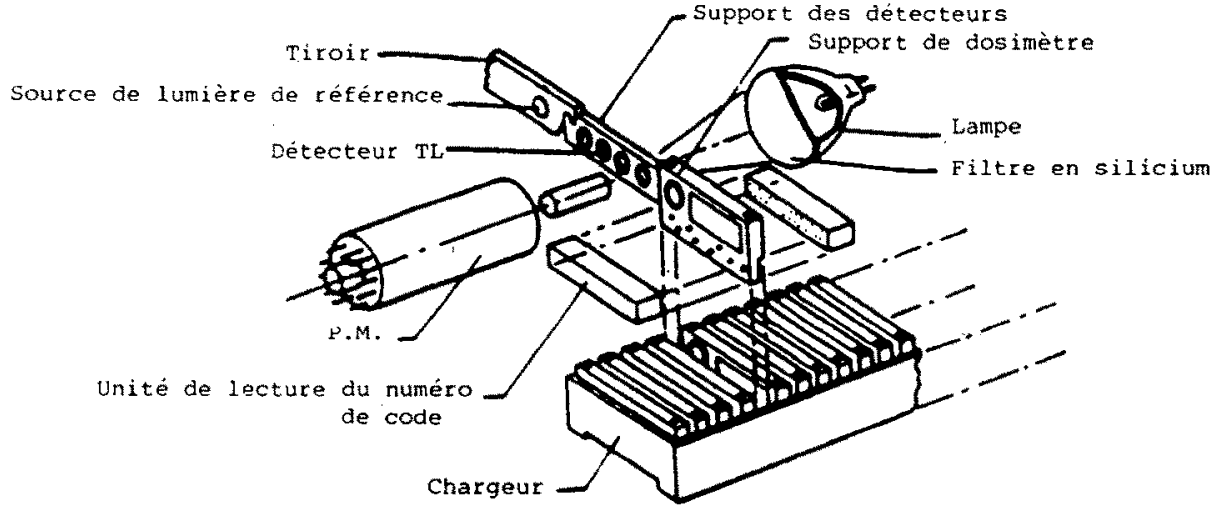

Fig. 11. - Panasonic à chauffage IR. Vue schématique. 
Le chauffage est effectué par le rayonnement (visible et IR) d'une lampe à halogène. Le rayonnement IR, seul utilisé pour chauffer, est séparé du rayonnement visible à l'aide d'un filtre en silicium de quelques micromètres d'épaisseur. Le PM et la source IR sont situés de part et d'autre de l'échantillon à mesurer. L'absence de contact thermique n'entraîne pas nécessairement une meilleure reproductibilité car l'évolution de l'émissivité du substrat absorbant porte-détecteur (côté source IR) varie, en général, avec le temps. Par contre, la durée du chauffage est très courte, moins d'une seconde.

\subsection{Lecteur TNO à jet d'azote chaud [8]}

Le principe même du chauffage à azote chaud $\left(400\right.$ à $\left.500^{\circ} \mathrm{C}\right)$ interdit l'emploi de la poudre. Comme pour le lecteur précédent, la régulation thermique est difficilement contrôlée. Il y a souvent un dépassement thermique entraînant une émission de corps noir non négligeable. La vitesse de chauffage est modeste, la séquence complète de lecture avoisine $20 \mathrm{~s}$. Les figures 12 et 13 présentent la conception de la tête de mesure avec 3 entrées d'azote chaud pour homogénéiser le chauffage du fritté.

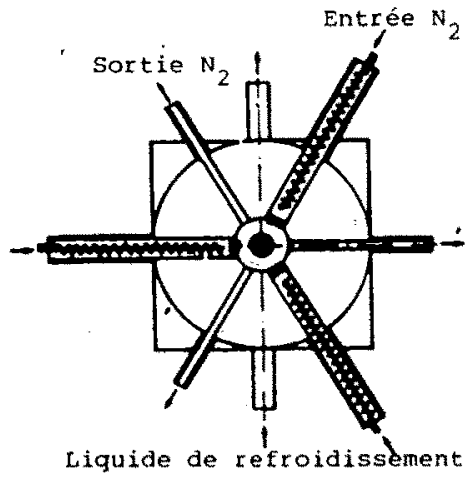

Fig. 12. - Lecteur à jet d'azote. Principe de mesure.

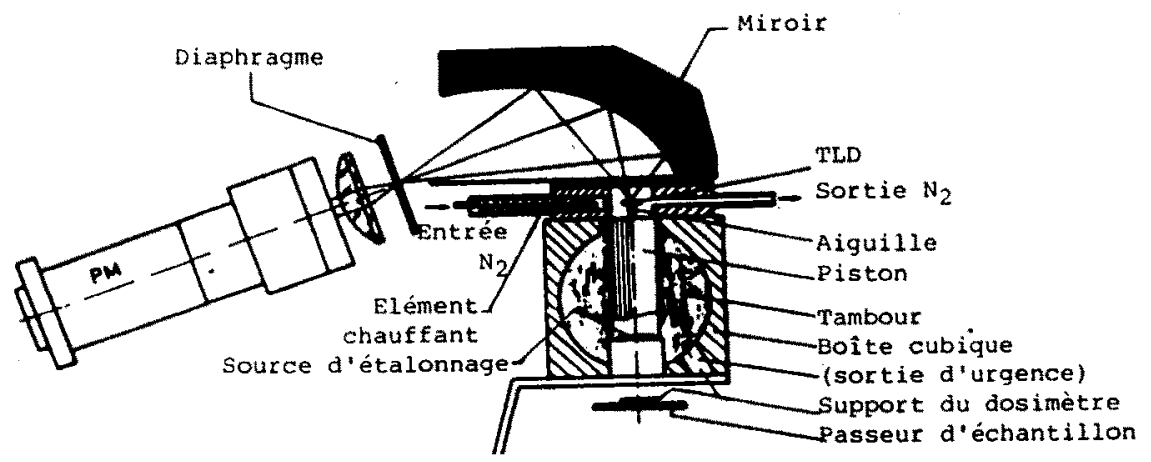

Flg. 13. - Lecteur a jet d'azote. Vue schómatlque. 


\subsection{Lecteur de International Sensor Technology (USA) [1]}

Ce lecteur mérite une attention toute particulière car il rompt avec les méthodes traditionnelles de chauffage. Présenté pour la première fois au congrès "Solid State Dosimetry" à Ottawa (Canada), 1983, son succès scientifique fut certain. Un laser à $\mathrm{CO}_{2}$, longueur d'onde : $10,6 \mu \mathrm{m}$; fréquence de répétition: $60 \mathrm{~Hz}$; puissance : $10 \mathrm{~W}$ et 0,3 à $2 \mathrm{~mm}$ de diamètre utile de surface chauffante suivant l'optique choisie pour l'élargissement du faisceau. La vitesse de chauffage est très grande pour les matériaux en couches minces (environ $10^{4} \mathrm{C} / \mathrm{s}$ ) ; la figure 14 montre le profil thermique surfacique, la figure 15 montre l'évolution de la zone TL en fonction du temps pour un chauffage faible $(4 \mathrm{~W})$ et un substrat épais $(1 \mathrm{~mm})$.

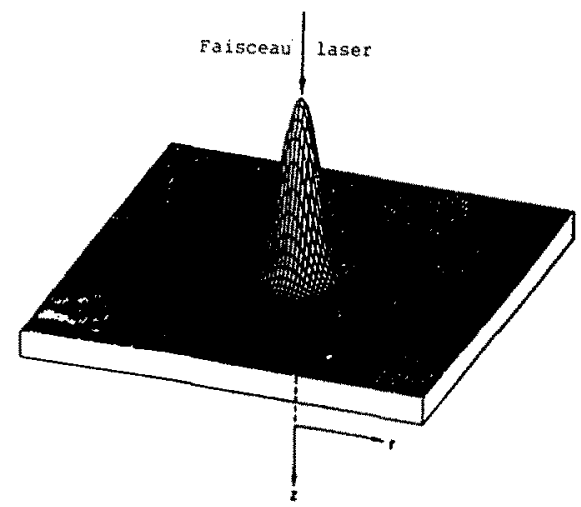

Fig. 14. - Profll thermique surfacique.

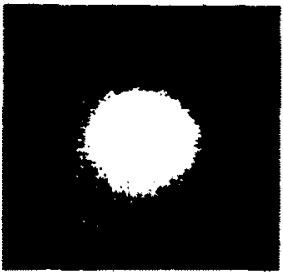

a

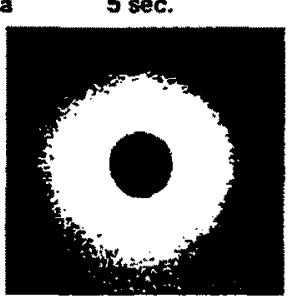

$c$ 22 sec.

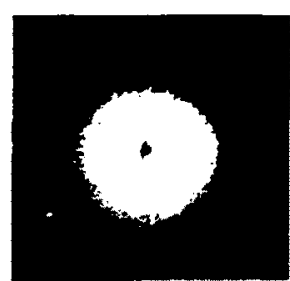

b

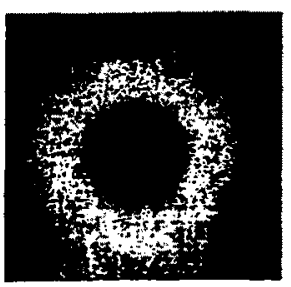

d $35 \mathrm{sec}$.

Fig. 15. - Image TL diffóronts Instants. 
Les figures 16 et 17 donnent l'évolution de l'intensité TL en fonction de la section du faisceau laser et du temps préchauffage à $100^{\circ} \mathrm{C}$ respectivement. La faible pente d'extinction est due à la diffusion thermique en surface qui engendre une TL parasite. Si tout l'échantillon est éclairé simultanément, on obtient une véritable impulsion de quelques centaines de millisecondes.

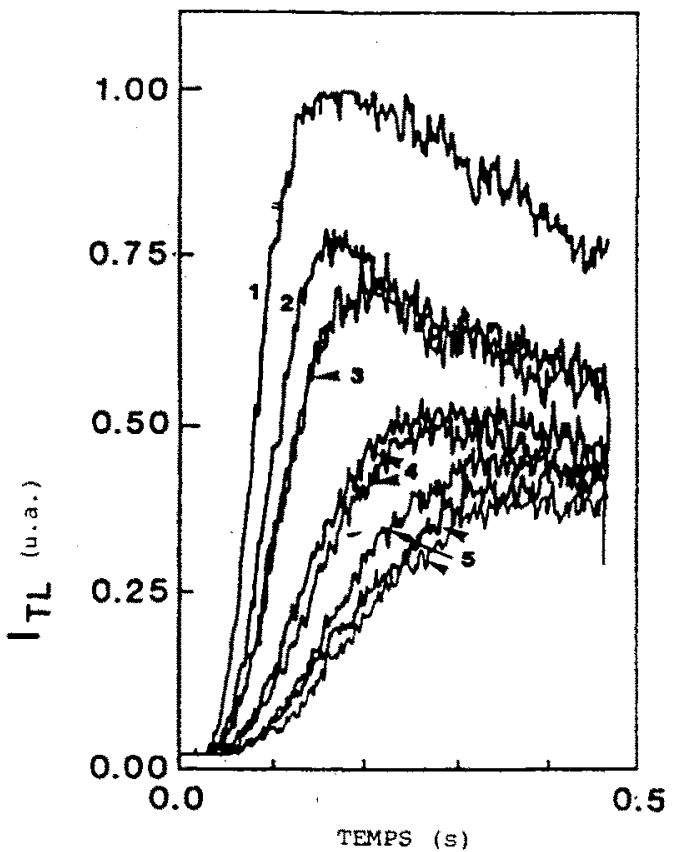

Flg. 16. - Intensité TL en fonction de la section du falsceau.

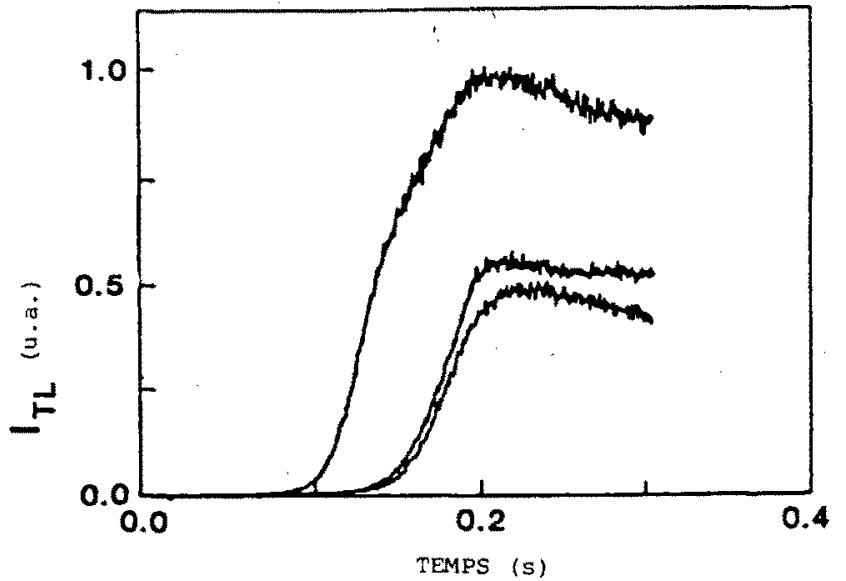

Fig. 17. - Effet d'un préchauffage a $100{ }^{\circ} \mathrm{C}$ pendant 10 secondes. 
La technique laser est surtout utilisée en imagerie TL avec une résolution de l'ordre du millimètre.

\subsection{Lecteur PCL (CEA) [3] (fig. 18)}

Le lecteur PCL a été développé pour permettre la mesure automatique de poudres et de différents frittés.

II comporte 5 postes principaux de travail et 5 postes intermédiaires. Un plateau rotatif permet de déplacer les coupelles dans un ordre donné sur les différents postes :

$1 /$ alimentation du plateau (coupelles empilées),

$2 /$ préchauffage isotherme (environ $180^{\circ} \mathrm{C}$ ),

$3 /$ lecture/chauffage isotherme $\left(500^{\circ} \mathrm{C}\right)$,

4/ évacuation ou nouveau cycle de lecture,

$5 /$ irradiation (radioprotection)

Cinq postes intermédiaires assurent les fonctions annexes :

$1 /$ contrôle de /alimentation en coupelles,

$2 /$ refroidissement transitoire,

$3 /$ choc thermique après la lecture,

$4 /$ contrôle de l'évacuation,

$5 /$ irradiation variable (longue durée en radiothérapie).

Une chambre à azote de grandes dimensions englobe les postes de préchauffage et de lecture permettant à la poudre d'éliminer, par diffusion, l'oxygène absorbé à la surface des grains. Le PM situé au-dessus du doigt de chauffage possède 3 filtres interchangeables suivant le type de matériau détecteur utilisé ; l'un d'eux peut être remplacé par une source lumineuse d'étalonnage. Le système de rechargement permet de récupérer les coupelles intactes (poudres ou frittés) dans l'ordre de lecture. Développé pour le borate de lithium, il permet d'atteindre "sans pesée" une précision voisine de $1 \%$ avec un temps moyen de lecture d'environ $15 \mathrm{~s}$ en lecture simple, $30 \mathrm{~s}$ avec lecture de la $1^{\text {ère }}$ résiduelle ou d'un étalonnage faible dose (10 mGy environ) et plusieurs minutes pour un étalonnage à forte dose avec un fort débit $(0,1 \mathrm{~Gy} / \mathrm{min})$.

Le lecteur est bâti autour d'un micro-ordinateur type PC 286 ; il comprend les éléments de base suivants :

$1 /$ l'ensemble mécanique et optique de lecture,

$2 /$ le rack électronique de contrôle et d'alimentation,

3/ l'ordinateur compatible PC (CPU 80286, $12 \mathrm{MHz}$, RAM : 1Mo, écran EGA, disque : $20 \mathrm{Mo}$, imprimante),

4/ la carte de commande spécialisée,

$5 /$ le logiciel de gestion à fenêtres et menus interactifs. 
La précision obtenue en routine (PCL1) est très voisine de $1 \%$ avec le $\mathrm{Li}_{2} \mathrm{~B}_{4} \mathrm{O}_{7}$ : $\mathrm{Cu}$. Les caractéristiques dosimétriques de la poudre étudiée conjointement par G. Marinello (CHU Créteil), R. Visocékas (Univ. P.-M. Curie) et $S$. Lorrain (CEA) sont décrites par $G$. Marinello [12].

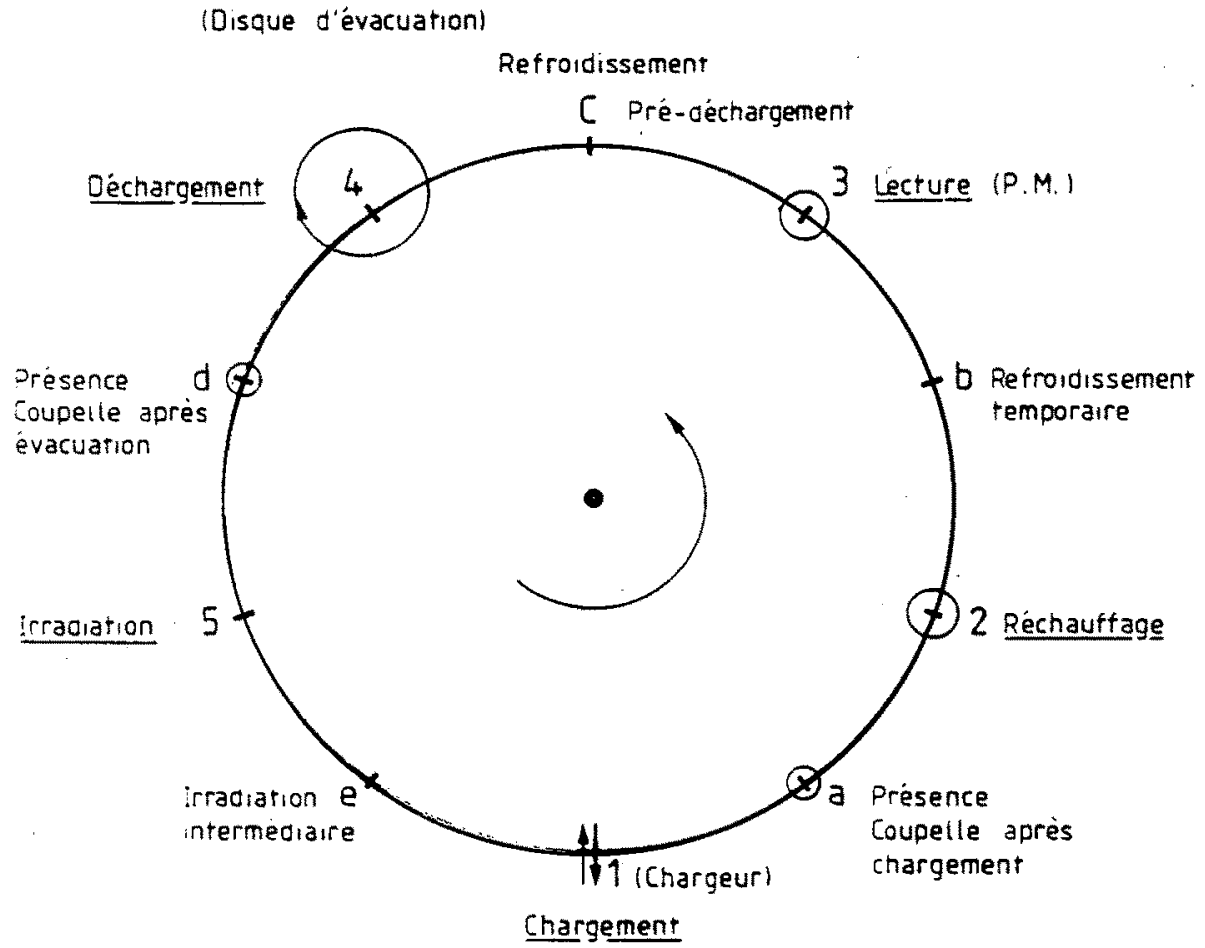

Fig. 18. - Schóma de princlpe du lecteur CEA PCL2.

\section{CONCLUSION}

La dosimétrie par thermoluminescence reste et restera encore pendant de nombreuses années une technique de choix pour la dosimétrie en radiothérapie. Avec les matériaux détecteurs actuels, en particulier le borate de lithium activé au cuivre, équivalent tissu presque parfait, et la nouvelle génération de lecteurs automatiques, la qualité, la reproductibilité et la rapidité d'obtention des résultats sont un atout important dans le suivi dosimétrique du patient [12]. 


\section{BIBLIOGRAPHIE}

[1] ABTAHI A., BRAUNLICH P., KELLY P., GASIOT J. - Laser stimulated thermoluminescence. J. Appl. Phys., 1985, 58 (4) 1626-1639.

[2] BARTHE J., BOHM J., CHRISTENSEN P., DRISCOLL C.M.H., HARVEY J.R., JULIUS H.W., MARSHALL M., MARSHALL T.O., OBERHOFFER M. - Report on a workshop on the application of the thermoluminescence dosimetry to large scale individual monitoring, Ispra 11-13 sept 85, Radiat. Prot. Dosim., 1987, 18 (1) $47-61$

[3] BARTHE J., PORTAL G., BLANCHARD Ph., et LAUREANA C. - Lecteur PCL1 et PCL2. Cahier des spécifications techniques $n^{\circ} 1$ et 2 (1983-1987). Fontenayaux-Roses : IPSN/DPT/SIDR.

[4] BERMANN F. - La Dosimétrie individuelle au Laboratoire d'exploitation dosimétrique de Fontenay-aux-Roses. Paris : CEA, Département des Relations publiques et de la communication, 1987.

[5] BIKWAKU N.T. - Dosimétrie par thermoluminescence en atmosphère très chaude en vue de la dosimétrie dans les réacteurs nucléaires, Diplôme d'études supérieures, $n^{\circ} 31 / 85$, Toulouse, octobre 1985.

[6] DAVID J.P. - Contrôles non destructifs de nouvelles préparations de borate de lithium. Application à la dosimétrie par thermoluminescence, Thèse de spécialité, Univ. Pierre et Marie Curie, Paris, décembre 1985.

[7] DUFTSCHMID K.E., LAUTERBACH U. et PATTISON R.J. - Comparison of most widely used automated TLD readout systems. EURADOS-CENDOS : Aspects of individual monitoring. Radiat. Prot. Dosim., 198614 (1), 33-39.

[8] JULIUS H.W. - Instrumentation In : Applied thermoluminescence dosimetry, Ispra Courses (M. OBERHOFER and A. SCHARMANN, Eds.) Bristol : Adam Hilger, $1981,39-66$.

[9] HOROWITZ Y.S. - Thermoluminescence and thermoluminescent dosimetry, volumes 1, 2, 3. Boca Raton, Florida : C.R.C. Press, 1984.

[10] LAPRAZ D., IACCONI Ph. - Principes de base et theorie de la thermoluminescence. In : Séminaire de biophysique médicale, Nice 22-23 févr. Radioprotection, 1990,25 (2) 117-133.

[11] MCKINLAY A.F. - Thermoluminescence dosimetry, medical physics handbooks. Bristol : Adam Hilger, 1983.

[12] MARINELLO G., POLLACK J., BLANCHARD Ph., BARTHE J. - Comparaison des conditions pratiques d'utilisation d'un lecteur à chauffage traditionnel et d'un lecteur automatique à chauffage rapide. In : Séminaire de biophysique médicale, Nice, 22-23 févr. Radioprotection, 1990, 25 (2) 157-167.

[13] M.T.O. - Informations techniques M.T.O., 11, rue Ampère, Massy, France, 1988.

[14] OBERHOFER M., SCHARMANN A., Eds. Applied thermoluminescence dosimetry, Ispra Courses. Bristol : Adam Hilger, 1981.

[15] PORTAL G. - Etude et développement de la dosimétrie par radiothermoluminescence, Thèse d'Etat $n^{\circ} 821$, Toulouse, juil. 1978 et Rapport CEA-R-4943, 1978. 